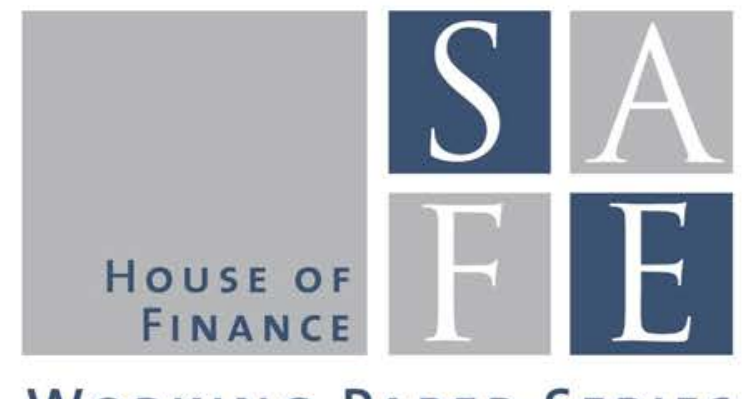

WORKING PAPER SERIES

Monica Billio - Massimiliano Caporin - Roberto Panzica - Loriana Pelizzon

\title{
The impact of network connectivity on factor exposures, asset pricing and portfolio diversification
}

SAFE Working Paper No. 166

SAFE I Sustainable Architecture for Finance in Europe A cooperation of the Center for Financial Studies and Goethe University Frankfurt 


\section{Non-Technical Summary}

The term systematic risk is a well-established concept that derives from works on portfolio choice, extended in a general equilibrium framework and in the Arbitrage Price Theory (APT) model. It refers to the risk to which an investor in a well-diversified portfolio is exposed, which stems from the dependence of the returns on common factors. However, the literature stressed that the determinants of systematic risk need to be analyzed with more detail. In this paper, we provide a unique framework for systematic risk and network connections, and estimate the feedback between network exposures and common factors and their impact on the factor risk exposures and risk premia of stock returns. We will show that by introducing into a multifactor asset pricing model the impact of contemporaneous links, that exist across assets, when those links are captured by a network. The network provide information on the existence of links and might also convey details of the intensity of the links existing between assets. Therefore, we aim to couple the systematic and idiosyncratic risks with a sort of network risk that would introduce into the model the assets' crossdependence, beyond that captured by common factors. Given this further element, we then evaluate the effects on traditional uses of the multifactor model. The network relations can be, in some sense, forward looking or represent the actual state of the connections across assets. We will demonstrate that the presence of interconnections implies that risky assets are exposed to the movements (both systematic and idiosyncratic) of other risky assets.

This extension allows a better understanding of the causes of systematic risk and shows that (i) network exposures act as an inflating factor for systematic exposure to common factors and (ii) the diversification power is reduced by the presence of network connections. Moreover, we show that in the presence of network links a misspecified traditional linear factor model presents residuals that are correlated and heteroskedastic. We support our claims with an extensive simulation experiment. 


\title{
The impact of network connectivity on factor exposures, asset pricing and portfolio diversification *
}

\author{
Monica Billio $^{\dagger} \quad$ Massimiliano Caporin $^{\ddagger} \quad$ Roberto Panzica $^{\S}$ \\ Loriana Pelizzon
}

First Version: May 2014

This version: October 2016

\begin{abstract}
This paper extends the classic factor-based asset pricing model by including network linkages in linear factor models. We assume that the network linkages are exogenously provided. This extension of the model allows a better understanding of the causes of systematic risk and shows that (i) network exposures act as an inflating factor for systematic exposure to common factors and (ii) the power of diversification is reduced by the presence of network connections. Moreover, we show that in the presence of network links a misspecified traditional linear factor model presents residuals that are correlated and heteroskedastic. We support our claims with an extensive simulation experiment.
\end{abstract}

Keywords: CAPM, volatility, network, interconnections, systematic risk.

JEL Classification: G10, G12, F35, C58.

\footnotetext{
*We gratefully acknowledge research support from the Research Center SAFE, funded by the State of Hessen initiative for research LOEWE. We thank the participants to the Computational and Financial Econometrics 2014 conference, the Systemic Risk and Contagion 2014 workshop in Bologna, the CREDIT2014 conference, for the comments provided. The authors acknowledge financial support from the European Union, Seventh Framework Program FP7/2007-2013 under grant agreement SYRTO-SSH2012-320270, from the MIUR PRIN project MISURA - Multivariate Statistical Models for Risk Assessment, and from Global Risk Institute in Financial Services and the Louis Bachelier Institute under the project Systemic Risk.

†University Ca’ Foscari Venezia (Italy)

$\ddagger$ University of Padova (Italy)

$\S \mathrm{SAFE} /$ Goethe University Frankfurt (Germany)

`SAFE/Goethe University Frankfurt (Germany)
} 


\section{Introduction}

The term "systematic risk" is a well-established concept that derives from the seminal work on portfolio choice proposed by Markowitz (1952) and extended in a general equilibrium framework by Sharpe (1964), Lintner (1965a, 1965b) and Mossin (1966), and in the Arbitrage Price Theory model by Ross (1976). It refers to the risk to which an investor in a well-diversified portfolio is exposed, which stems from the dependence of the returns on common factors. However, as stressed by Cochrane (2011), there is a need for a better understanding of the determinants of systematic risk.

In this paper we provide a unique framework for systematic risk and network connections, and estimate the feedback between network exposures and common factors and their impact on the factor risk exposures and risk premia of stock returns.

A growing literature investigates the role of interconnections between different firms and sectors as a potential mechanism for the propagation of shocks throughout the economy. Acemoglou et al. (2012) use network structure to show the possibility that aggregate fluctuations may originate from microeconomic shocks to firms. Kelly et al. (2013) show how stock firm volatility is related to customer-supplier connectedness. Billio et al. (2014) use contingent claim analysis and network measures to highlight interconnections between sovereigns, banks and insurance. There are several other contributions to the literature on network analysis: see Billio et al. (2012), Diebold and Yilmaz (2015), Hautsch, Schaumburg and Schienle (2012, 2013), Barigozzi and Brownlees (2014) and Ozdagli and Weber (2015), Fernandez (2011), Kou et al. (2016). Network interconnections and the effects called network externalities that arise from small and local shocks that can become big and global are a possibility discarded in standard asset pricing and macroeconomic models due to a "diversification argument". As argued by Lucas (1977), among others, microeconomic shocks will average out and thus have only negligible aggregate effects. Similarly, they will have little impact on asset prices. However, there is also a growing literature on the role of sectorial shocks in macro fluctuations. Examples include Horvath (1998), Dupor (1999), Shea (2002) and Acemoglu et al. (2012). Moreover, Ang et al. (2006), among 
others, show that idiosyncratic volatility risk is priced in the cross-section of expected stock returns, a regularity that is not subsumed by size, book-to-market, momentum or liquidity effects. From a theoretical point of view, Wagner (2010), Ozsoylev and Walden (2011), Buraschi and Porchia (2013) and Branger et al. (2014) arrive at similar conclusions. Ahern (2013) empirically documents a positive market price of centrality, i.e., that more central assets earn higher expected returns.

The contribution of this paper to this literature is to propose a modelling framework in which network interconnections and common factor risks co-exist. The proposed model is a variation on the traditional Capital Asset Pricing Model (CAPM) or Arbitrage Pricing Theory (APT) framework in which networks are used to infer the exogenous and contemporaneous links across assets. We are able to disentangle direct exposures of a single stock to common factors from the indirect exposure to the common factors that arise from network interconnections. We also provide a number of generalizations to our approach to make it more flexible and coherent with the empirical evidence, for instance allowing for asset-specific reaction to network links and introducing time variation into networks.

By building on the newly introduced model, we provide a number of theoretical elements and pieces of empirical evidence based on a simulation framework. First, focusing on the dynamics of returns and the exposure to common factors, we show that the presence of asset interconnection acts to inflate the exposure to common risk sources. Moreover, we are able to disentangle the exposure to common factors that is structural, which is present even in the case of no network connections, from the exposure associated with network links. A similar argument applies to the shocks impacting on an asset return, whereby network relations expose assets to other assets' shocks. From a risk perspective, our approach allows us to decompose the risk of a single asset (or a portfolio) into four components: (i) the systematic component, (ii) the idiosyncratic component, (iii) the impact of the asset interconnections on the systematic risk component, that is, the contribution of network exposure to the systematic risk component, and (iv) the effect of interconnections between the idiosyncratic risks on the systematic risk component, that 
is, the amplification of idiosyncratic risks that generates systematic/non-diversifiable risk. Building on this result, we show how diversification benefits are reduced in the presence of network connections. Moreover, by combining the return dynamics with the variance decomposition, we can verify that our model is consistent with the presence of correlation and heteroskedasticity among traditional linear-factor-model residuals, thus providing a rationale for the empirical evidence found in the literature.

The simulation analysis allows us to disentangle the error estimation of linear factor models that ignore the presence of network connections. In particular we show that, when asset returns are significantly related to network interconnections, the factor loading estimation of common factors is largely misspecified if the estimation is based on a traditional linear factor model. Moreover, the residuals' correlations start drifting away from zero if network connections are ignored in the model estimation.

Finally, we also evaluate the impact of networks on the estimation of risk premiums and show that the premiums estimated by our approach and by a traditional linear factor model are equivalent in the long run (under some assumptions on the evolution of the network over time). However, our approach allows for local (conditional) expected returns that change according to changes in the network structure, and thus lead to price changes even if the risk premiums are time-invariant.

The remainder of the paper is organized as follows. Section 2 describes network models. Section 3 presents our model combining network links and factor exposure, while Section 4 introduces a set of generalizations making the model more flexible. Section 5 describes the estimation methodology for the model augmented with the network links. Section 6 presents the simulation analysis and finally Section 7 concludes. 


\section{Network Models in Finance}

\subsection{Review of the literature}

Network models have featured in an extremely diverse array of applications: in the social sciences with studies related to social networking on websites such as Facebook, in the natural sciences with application to protein interactions, in government intelligence where they are used to analyse terrorist networks, in politics with application to bill coauthorship, in economics with the potential to be used in labour market analysis, and many other areas. In finance, network models have most frequently been used to assess financial stability. In fact, interconnections between financial institutions create potential channels for contagion and the amplification of shocks to the financial system that can also propagate into the "real economy". Theoretical and empirical studies in this area have garnered considerable interest in the aftermath of the 2007-2009 financial crisis. Network representation of interconnections ranges from linkages extracted from balancesheet information to connections estimated by means of econometric approaches based on market, accounting or macroeconomic data.

The majority of such "real-world" networks have been shown to display structural properties that are neither those of a random graph, nor those of regular lattices.

In order to evaluate the relevance and the price of interconnections in the financial system it is fundamental to understand all of the channels by which small and local shocks can become big and global.

Empirical network modelling has been conducted to assess asset pricing linkages via contagion (Allen and Gale, 2000; Dasgupta, 2004; Leitner, 2005; Billio et al., 2012; Bianchi et al., 2015; Diebold and Yilmaz, 2014; Hautsch, Schaumburg and Schienle, 2012, 2013.), linkages via balance sheets (Cifuentes, Ferrucci and Shin, 2005; Lagunoff and Schref, 2001) and how failures of institutions result from mutual claims on each other (Furfine, 2003; Upper and Worms, 2004; Wells, 2004; Billio et al., 2014; Ozdagli and Weber, 2015). Allen and Babus (2009) provide a review of network models in finance. 
Much of the empirical finance literature has focused on "direct" contagion arising from firms' contractual obligations. Direct contagion occurs if one firm's default on its contractual obligations triggers distress (such as insolvency) at a counterparty firm. Researchers' simulations using actual interbank loan data suggest that "domino defaults" arising from contractual violations are very unlikely (see Furfine, 2003; Elsinger, Lehar and Summer, 2006; Upper and Worms, 2004; Mistrulli, 2011; Degryse and Nguyen, 2007; Van Lelyveld and Liedorp, 2006; Alves et al., 2013), though they can be highly destructive in the event that they do materialize.

\subsection{Formal representation of networks}

Formally, we could represent networks as nodes that are connected (in general) to a subset of the total number of nodes in the network, in which connections represent links across nodes. A financial system could be represented as a network structure in which nodes represent assets or the value of financial or non-financial institutions, and shocks on one asset/institution are transmitted to those connected to it.

Networks are, in general, graphically represented. Nevertheless, networks have an equivalent (square) matrix representation. Let us call $W$ the $K$-dimensional square matrix representing a network composed of $K$ financial assets/companies. Each entry $w_{i, j}$ represents the possible connection between assets $i$ and $j$. A zero entry indicates that the two assets are not connected, while a non-null entry indicates the existence of a connection. Depending on the approach adopted to estimate the network, non-null entries might differ from one another, tracking the strength/intensity of the connection, or be equal to one another, simply indicating the existence of a connection. An example of the latter case is the following matrix: 


$$
W=\left[\begin{array}{lllll}
0 & 1 & 0 & 1 & 0 \\
1 & 0 & 1 & 0 & 0 \\
0 & 1 & 0 & 1 & 0 \\
0 & 0 & 1 & 0 & 1 \\
0 & 0 & 0 & 1 & 0
\end{array}\right],
$$

where it should be noted that the diagonal contains only null elements (no asset influences itself) and that the network is not symmetric as the first asset is connected to the fourth, but the opposite is not true. In general, networks also convey a further element, the direction of the link. If links are all bidirectional, the network is symmetric. By convention, in the present paper we assume that a non-null element $w_{i, j}$ indicates the existence of a link between assets $i$ and $j$ with an effect from $j$ to $i$.

Interestingly, matrices similar to that given in equation (1) are very common in other economic and statistical applications, those concerning research and studies associated with spatial econometrics and spatial statistics. In these fields, subjects (such as towns, buildings or regions) are neighbours of each other in a physical way, and the matrix $W$ represents the neighbouring relations, with entries possibly associated with the physical distance existing between two subjects; such matrices are normally called spatial matrices, and are commonly row-normalized.

Matrix representation of financial networks might thus be seen as the financial parallel of spacial matrices. Clearly, neighbouring relations are no longer physical, but are the outcome of a specific model, measurement or estimation approach. Going back to the graphical representation of networks, in which nodes are connected to one another, we might state that connected nodes (assets/firms) are thus neighbours. Finally, we stress that, if we consider matrices to monitor only the existence of connections across assets, we adhere to the concept of "first-order contiguity" whereby a unit entry denotes the existence of a connection and the fact that two assets are neighbours (see LeSage, 1999). 
In addition, by convention in spatial statistics/econometrics, the main diagonal of the matrix $W$ contains zero elements.

In the following, we will clarify how network connections, as monitored by the matrix $W$, will convey relevant information on the evolution of asset returns. In doing so, we do not restrict ourselves to a specific structure of $W$, that is, a $W$ that monitors the existence of a connection and/or the intensity of the link, but propose a model that can be used with any form of $W$. Moreover, following Elhorst (2003), we normalize $W$ so that, if we are monitoring only the existence of the connection, we equalize the impact of each unit on all other units. We discuss the normalization of $W$ further in a later section. In the empirical part of the paper, we also briefly discuss alternative methods that can be used to estimate the existence of a connection between two assets.

\section{The Systematic Effects of Network Exposure}

\subsection{The classic framework}

Ever since the publication of the seminal works of Sharpe (1964), Lintner (1965a, 1965b), and Mossin (1966), linear returns models have attracted huge interest in the financial economics literature, and have had an extraordinary impact on both research and practice. In the last few decades, multifactor generalizations of the CAPM model have been proposed and are now as widespread as the single-factor model. The first multifactor model stems from the work of Ross (1976) on the APT, and the most commonly used approaches to pricing now take into account the developments of Fama and French (1993, 1995) and Carhart (1997), leading to the so-called three-factor and four-factor CAPM models, respectively.

Our starting point is a multifactor model, within which all the previous cases are nested, and which we take as a general case into which network exposures can be introduced. We thus consider a linear specification in which a $K$-dimensional set of time- $t$ risk 
asset returns, which we denote by $R_{t}$, depends on a set of $M$ observable zero-mean risk factors $F_{t}$ :

$$
R_{t}=\alpha+\beta F_{t}+\varepsilon_{t}
$$

In equation (2), $\alpha$ is a $K$-dimensional vector of intercepts, $\beta$ is a $K \times M$ matrix of parameters monitoring the exposure of the risky assets to the common factors included in the $M$-dimensional vector $F_{t}$, and $\varepsilon_{t}$ is the vector of idiosyncratic shocks.

If we take a pricing perspective, and assume that the market is in equilibrium, then the model intercept can be replaced by the vector of expected returns

$$
R_{t}=\mathbb{E}\left[R_{t}\right]+\beta F_{t}+\varepsilon_{t}
$$

Moreover, expected returns depend on the factor risk premiums $\Lambda$ satisfying

$$
\mathbb{E}\left[R_{t}\right]=r_{f}+\beta \Lambda
$$

The multifactor model allows for the decomposition of the total risk of the assets into the sum of two components: ${ }^{1}$

$$
\mathbb{V}\left[R_{t}\right]=\beta \Sigma_{F} \beta^{\prime}+\Omega_{\varepsilon}
$$

where $\mathbb{V}[\cdot]$ is the variance operator, $\mathbb{V}\left[F_{t}\right]=\Sigma_{F}$ is the covariance matrix of the common factors, and $\mathbb{V}\left[\varepsilon_{t}\right]=\Omega$ is the covariance matrix of the idiosyncratic shocks. The first term on the right represents the systematic contribution to the total risk, while the second term is the idiosyncratic risk contribution. The same decomposition of the total asset risk also applies to a generic portfolio formed using the $K$ assets. If we take a vector of portfolio weights $\omega]^{2}$ the portfolio returns satisfy the following equalities:

\footnotetext{
${ }^{1}$ This holds for any multifactor model.

${ }^{2}$ We assume that the portfolio weights sum to 1 but we do not exclude short selling.
} 


$$
\begin{aligned}
r_{p, t} & =\omega^{\prime} R_{t} \\
& =\omega^{\prime} \mathbb{E}\left[R_{t}\right]+\omega^{\prime} \beta F_{t}+\omega^{\prime} \varepsilon_{t} \\
& =\mathbb{E}\left[r_{p, t}\right]+\beta_{p} F_{t}+\varsigma_{t},
\end{aligned}
$$

where $\mathbb{E}\left[r_{p, t}\right]=r_{f}+\beta_{p} \Lambda$. Moreover, we know that the total risk of the portfolio is given by

$$
\begin{aligned}
\mathbb{V}\left[r_{p, t}\right] & =\omega^{\prime} \beta \Sigma_{F} \beta^{\prime} \omega+\omega^{\prime} \Omega_{\varepsilon} \omega \\
& =\beta_{p} \Sigma_{F} \beta_{p}^{\prime}+\sigma_{\varsigma}^{2} .
\end{aligned}
$$

This framework has relevant implications for portfolio risk and diversification. If we take a diversification point of view, the final purpose is to control or sterilize the impact of idiosyncratic asset risks on the total portfolio risk. This corresponds to the willingness of achieving the following limiting condition:

$$
\lim _{K \rightarrow \infty} \omega^{\prime} \Omega_{\varepsilon} \omega=\tilde{\sigma}^{2}>0
$$

where $\tilde{\sigma}^{2}$ is a small quantity depending on the idiosyncratic shock variances and correlations, as well as on the portfolio composition. In a simplified setting, assuming that idiosyncratic shocks are uncorrelated, that their variances are set to an average value $\bar{\sigma}^{2}$ and taking an equally weighted portfolio, we have the following well-known result:

$$
\lim _{K \rightarrow \infty} \omega^{\prime} \Omega_{\varepsilon} \omega=\frac{1}{K} \bar{\sigma}^{2}=0,
$$

showing that diversification allows the idiosyncratic shocks to be sterilized.

In this framework, the focus is on the shocks' impact, since we know that the systematic risk component cannot be diversified out, as it is driven by common factors. Therefore, in the multifactor model, the introduction of new assets allows a contraction 
of the contribution of the idiosyncratic component to the total risk of the portfolio but has, on average, no effects on the systematic components.

\subsection{Introducing network exposure}

Our paper aims at introducing into a multifactor asset pricing model the impact of contemporaneous links that exist across assets, when those links are captured by a network. As discussed in the previous section, networks provide information on the existence of links and might also convey details of the intensity of the links existing between assets. Therefore, we aim to couple the systematic and idiosyncratic risks with a sort of network risk that would introduce into the model the assets' cross-dependence, beyond that captured by common factors. Given this further element, we then evaluate the effects on traditional uses of the multifactor model.

Let us assume that the risky assets are interconnected and that those links can be represented by a network. The network relations, as observed in the previous section, can be, in some sense, forward looking or represent the actual state of the connections across assets. From this point onward, we assume that the network will impact on the contemporaneous relations across assets. We will show that the presence of interconnections implies that risky assets are exposed to the movements (both systematic and idiosyncratic) of other risky assets. Moreover, we will highlight how risky assets might differ in terms of interconnections with other assets. This creates an additional form of heterogeneity, going beyond those associated with the different exposures to common risk factors and with the different degrees of relevance of idiosyncratic shocks. Starting from this assumption, we reconsider the interpretation of a general multifactor model. If we postulate about the existence of contemporaneous relations across risky assets, we must acknowledge that such relations are not explicitly accounted for in equation (2). As a consequence, the beta matrix with respect to common factors that can be recovered from a traditional linear factor model, i.e. that of equation (2), cannot be directly linked to both the interconnections and the source of network heterogeneity across risky assets. 
One possible way of reconciling the model in equation (2) with the network exposure is to interpret the model as a reduced-form model whose reduced-form parameters (the betas and the error covariance) are functions of structural parameters' network exposure and structural factors such as macro factors etc. The latter thus include the true exposure to common structural factors, the exposure to other assets due to the interconnections (or network exposure) and the structural idiosyncratic shock's variance.

To shed some light on the previous points we rewrite the model in equation (3) as a structural simultaneous equation system (later on called a structural model),

$$
A\left(R_{t}-\mathbb{E}\left[R_{t}\right]\right)=\bar{\beta} F_{t}+\eta_{t},
$$

where the matrix $A$ captures the contemporaneous relations across assets and coexists with the common factors, which are here considered as exogenous variables. In equation (10) the covariance of $\eta_{t}$ represents the structural idiosyncratic risk while the parameter matrix $A$ is associated with assets' interconnections, and thus with a network. Further details on the latter aspect will be given in a few paragraphs' time. If we translate the model in equation 10 into a reduced form, we have

$$
\begin{aligned}
R_{t} & =\mathbb{E}\left[R_{t}\right]+A^{-1} \bar{\beta} F_{t}+A^{-1} \eta_{t} \\
& =\mathbb{E}\left[R_{t}\right]+\beta^{\star} F_{t}+\epsilon_{t}^{\star},
\end{aligned}
$$

where $\beta^{\star}$ is the matrix of reduced-form betas, equal to $\beta^{\star}=A^{-1} \bar{\beta}$ and $\epsilon_{t}^{\star}$ is the vector of reduced-form errors $3^{3}$ We stress two relevant elements. Firstly, we observe that the reduced-form parameters $\beta^{\star}$ are non-linear functions of the interconnections between assets (the matrix $A$ ) and of the structural exposure to common structural factors (the matrix $\bar{\beta}$ ). Secondly, the covariance matrix of the errors $\epsilon_{t}^{\star}$ is influenced by the presence of interconnections between assets.

\footnotetext{
${ }^{3}$ We include the star as a suffix to distinguish the reduced-form errors implied by our model from the errors of the traditional multifactor model.
} 
We postulate that a network structure exists, thus assets are interconnected and at the same time the assets depend on a common set of risk factors. If on these assets we estimate the linear factor model in equation (2) without taking the network into account, we have, by construction, that the shocks are correlated ${ }^{4}$ This is a consequence of the fact that the reduced-form betas can be estimated consistently in equation (11) anyway by standard linear regression models, but the residuals' covariance is not coherent with the theoretical expectation, that is, idiosyncratic shocks are uncorrelated. Therefore, the empirical evidence of idiosyncratic shock correlation found in the residuals of multifactor models might be due to the exclusion of contemporaneous relations. This is also coherent with the following finding of Ang et al. (2006): idiosyncratic volatility risk is priced in the cross-section of expected stock returns, a regularity that is not subsumed by size, book-tomarket, momentum or liquidity effects. In addition, if we assume that the network links affect the matrix $A$, and estimate the model in equation (11), the residuals' covariance will be a function of the network links. ${ }^{5}$ So far our analysis concerns only observable factors. If the common factors are estimated by means of statistical approaches rather than being observed variables, the network exposure, if present, will be totally destroyed. In fact, statistical factors are generally estimated from a reduced-form model. Therefore, if we adopt principal component analysis, or fit a latent factor model, it might happen that one of the identified factors represents a sort of proxy or biased estimate of the network exposure, with possible further biases on the estimated factor loadings. Such a problem might be overcome by estimating a latent factor model accounting for contemporaneous links across assets. Examples can be found in the studies of Barigozzi and Brownlees (2014) and Bianchi et al. (2015), who show that, if network links are not known, they might be estimated by looking at the covariance of $A^{-1} \eta_{t}$. However, in such a case, the economic interpretation of network links might be difficult to recover and could be

\footnotetext{
${ }^{4}$ This holds if we assume that $A$ is not diagonal. However, this is an inconsequential restriction as, if $A$ is diagonal, we do not have contemporaneous relations between assets.

${ }^{5}$ Assuming normality for the model innovations $\eta_{t}$, such that $\eta_{t} \sim N(0, \Omega)$, we have that the reducedform residuals follow the distribution $N\left(0, A(W)^{-1} \Omega_{n} A(W)^{-1}\right)$, where the reduced residuals are $\epsilon_{t}^{\star}=$ $A(W)^{-1} \eta_{t}$
} 
exposed to estimation error. In addition, the common factors we choose in the model must be exogenous. Our approach aims at reintroducing contemporaneous relations into the multifactor model, thus allowing both the impact of network exposure and the direct exposure to common structural factors to be recovered. Note that the two elements coexist, and network exposure can be seen as an additional common risk source going beyond that of common factors. We might even define the exposure to common factors as the exogenous systematic risk exposure, while the network exposure can be labelled as an endogenous systematic risk exposure. Further, we stress that we label the $\eta_{t}$ in equation (10) as structural idiosyncratic shock to distinguish it from the vector $\varepsilon_{t}^{\star}$ in equation (11) that represents the reduced-form idiosyncratic shock. The simultaneous equation system in equation 10 poses serious challenges for the estimation of the matrix $A$. We overcome this potential problem by resorting to network links. If we postulate the existence of network connections, exogenously provided by direct exposures and indirect, we can state that linked assets can easily be recast in a proximity matrix $W$ as mentioned in Section 2. The proximity matrix can be used to impose a structure on the matrix $A$. Given the matrix $W$, as extracted from a network, we can easily specify a spatial autoregressive (SAR) model (see Anselin, 1988; LeSage and Pace, 2009):

$$
R_{t}-\mathbb{E}\left[R_{t}\right]=\rho W\left(R_{t}-\mathbb{E}\left[R_{t}\right]\right)+\bar{\beta} F_{t}+\eta_{t}
$$

where the (scalar) coefficient $\rho$ captures the response of each asset to the returns of other assets, as weighted by the corresponding row of $W$. Moreover, we assume that the error term $\eta_{t}$ has a diagonal covariance matrix, that is, $\mathbb{V}\left[\eta_{t}\right]=\Omega_{\eta}$ is diagonal. Such an assumption is required for identification purposes, as we will discuss in the model estimation section. If we assume, as we will do in the following, that the matrix $W$ is known, the expected returns are conditional upon the (known) $W .7$

\footnotetext{
${ }^{6}$ Anselin (1988) calls the model mixed-regressive spatial-autoregressive. We stick here to the simpler acronym adopted in LeSage and Pace (2009).

${ }^{7}$ To maintain a simplified notation, we do not report the conditioning with respect to $W$ in the returns expectations.
} 
At the single asset level, the model reads as follows:

$$
R_{i, t}=\mathbb{E}\left[R_{i, t}\right]+\rho \sum_{j=1}^{k} w_{i, j}\left(R_{j, t}-\mathbb{E}\left[R_{j, t}\right]\right)+\bar{\beta}_{i} F_{t}+\eta_{i, t}
$$

where $w_{i, i}=0, w_{i, j} \geq 0$ and $\sum_{j=1}^{k} w_{i, j}=1$. Taking a financial point of view, the coefficients in the vector $\bar{\beta}_{i}$ represent the exposure to the common factors, or exogenous exposure, while the coefficient $\rho$ tracks the endogenous risk exposure, which is influenced by the network structure and is thus called network exposure. Further insights on the interpretation of the model coefficients will be given in the following subsections.

The model in equation $(12)$ can be rewritten in a more compact form as follows:

$$
(I-\rho W)\left(R_{t}-\mathbb{E}\left[R_{t}\right]\right)=\bar{\beta} F_{t}+\eta_{t}
$$

thus highlighting the fact that spatial proximity and the associated SAR model give a structure to the contemporaneous relation matrix, which is now parametrized as

$$
A=I-\rho W
$$

The structural model now includes contemporaneous relations, driven by links or connections across assets, systematic components and asset-specific shocks. We now elaborate on the relation between returns, risk, networks and risk factors.

\subsection{Returns, networks and risk factors}

The reaction of one asset to common factors and network exposure becomes clearer once we rewrite the model in a reduced-form representation. In this way, we highlight the impact of the network connections included in $W$ on the reduced-form parameters (the reduced-form betas and the reduced-form shock's covariance). The reduced-form model 
reads as in equation (11) with $A=I-\rho W$ now:

$$
\begin{aligned}
R_{t} & =\mathbb{E}\left[R_{t}\right]+(I-\rho W)^{-1} \bar{\beta} F_{t}+(I-\rho W)^{-1} \eta_{t} \\
& =\mathbb{E}\left[R_{t}\right]+\beta^{\star} F_{t}+\epsilon_{t}^{\star},
\end{aligned}
$$

where the factor loadings equal $\beta^{\star}=(I-\rho W)^{-1} \bar{\beta}$ and for the moment we assume that $A=I-\rho W$ is non-singular 8 For simplicity, we focus on the case in which the network exposure is driven by a single parameter, $\rho$. However, all derivations and comments also apply to the more general parametrizations of the matrix $A$ that we will introduce in Section 4 .

From LeSage and Pace (2009) we take the following relation:

$$
(I-\rho W)^{-1}=I+\rho W+\rho^{2} W^{2}+\rho^{3} W^{3} \ldots,
$$

where the term $\rho W$ monitors the effect of linked assets (in spatial econometrics, the neighbours). For instance, if asset $j$ is linked to asset $i$ we have a non-null entry in $W_{i j}$. Differently, $\rho^{2} W^{2}$ is associated with the effect on asset $j$ induced by the assets linked to asset $i$ (in spatial econometrics called the second-order neighbours). The latter relation can be further generalized to higher orders. Notably, the matrices $W^{j}$ might also include a so-called feedback loop as, following the previous example, asset $i$ can be linked to asset $j$ (making the relation bidirectional), causing the matrix $W^{j}$ to have non-null elements on the main diagonal. We stress that, despite the fact that the summation has an infinite number of terms, by imposing that $|\rho|<1$ we can easily ensure that the effect of linked assets converges to a finite number. On the contrary, if $|\rho|>1$ we might have explosive patterns. In general, the coefficient $\rho$ takes values in the range $\left(\lambda_{\min }^{-1}, \lambda_{\text {max }}^{-1}\right)$, with $\lambda_{\min }$ and $\lambda_{\max }$ respectively the minimum and maximum eigenvalues of $W$. In the case of rownormalization of the $W$ matrix, in spatial econometrics a commonly adopted range is

\footnotetext{
${ }^{8}$ See the model estimation section for details on the constraints that ensure non-singularity of $A$.
} 
$[0,1)$

Using equation (18), we can rearrange the model in equation (17) as

$$
R_{t}=\mathbb{E}\left[R_{t}\right]+\bar{\beta} F_{t}+\sum_{j=1}^{\infty} \rho^{j} W^{j} \bar{\beta} F_{t}+\eta_{t}+\sum_{j=1}^{\infty} \rho^{j} W^{j} \eta_{t}
$$

Such a representation highlights that the impact of the common factors as well as that of the idiosyncratic shocks on the risky asset returns can be decomposed into two parts. For both elements, the first component is the traditional, or direct, or structural impact of the structural common factor, while the second component is the impact associated with the network exposure. We can thus define the following four elements:

a) $\bar{\beta}$ : the structural exposure to the structural common factor;

b) $\sum_{j=1}^{\infty} \rho^{j} W^{j} \bar{\beta}$ : the network exposure to the structural common factor;

c) $\eta_{t}$ : the idiosyncratic shocks;

d) $\sum_{j=1}^{\infty} \rho^{j} W^{j}$ : the network impact of idiosyncratic shocks.

Note that the network-related exposures depend on the structure of the matrix $W$ as well as on the parameter monitoring the network impact, $\rho$. A relevant remark can be made in relation to the network impact of common factors. Let us take, for simplicity, a specific common factor. That is, we focus on a single column of $F_{t}$ and consider the impact of the $m$-th factor on the risky asset returns:

$$
\beta_{m}^{\star}=\bar{\beta}_{m}+\sum_{j=1}^{\infty} \rho^{j} W^{j} \bar{\beta}_{m}
$$

Starting from the observation that the reduced-form betas equal the sum of two elements, equation (20) provides two relevant insights.

First, we note that the network exposure to common factors acts as a multiplier of the structural exposure if the coefficient $\rho$ is positive ( $W$ 's elements are positive anyway). 
Therefore, shocks to the common factors will be amplified by (i) the presence of connections across assets, that is, when, for asset $i$, the $i$-th row of $W$ contains at least one non-null element, (ii) the change in the impact of network connections, that is, when the coefficient $\rho$ increases, and (iii) by changes in the network structure, that is, when the matrix $W$ changes. Note that, if asset $i$ is not connected to other assets, all products $\rho^{j} W^{j} \bar{\beta}_{i}$ are equal to zero.

From a different viewpoint, the presence of network exposure allows us to decompose the reduced-form betas into two components, a structural one and a multiplier depending on the network structure, the matrix $W$. The estimation of a standard factor model, where the data-generating process includes network dependence across returns, will provide partial information, returning only the combination of the two components, that is, only the reduced-form betas.

Now assume that, for the risky asset $i$, the $m$-th common factor is not relevant (that is, $\left.\bar{\beta}_{i, m}=0\right)$. In this case, in the standard linear factor models, the common factor will have no role in explaining the asset returns. However, when assets are linked and network exposures are taken into account, a common factor to which a risky asset has zero structural exposure might still be relevant for explaining the evolution of the risky asset return. Such an effect is not direct but induced from the network exposure and is associated with the existence of non-null elements in the $i$-th row of the matrix $W$. Take, for instance, the following case:

$$
W=\left[\begin{array}{ccc} 
& \vdots & \\
& & \\
\mathbf{0}_{i} & 1 & \mathbf{0}_{K-i-1} \\
& \vdots &
\end{array}\right]
$$

where asset $i$ is connected only to asset $i+1$ and the subscripts denote the lengths of the row vectors of zeros. Moreover, assume the following factor exposure for both assets: 


$$
\bar{\beta}=\left[\begin{array}{cccc} 
& \vdots & & \\
\bar{\beta}_{1, i} & 0 & 0 & 0 \\
\bar{\beta}_{1, i+1} & \bar{\beta}_{2, i+1} & 0 & 0 \\
& \vdots & &
\end{array}\right],
$$

where, in a multifactor model, asset $i$ is not exposed to factor 2 , while asset $i+1$ is affected by the same risk factor, and both assets are exposed to factor 1. Asset $i$ 's dependence on risk factors can thus be represented as

$$
\bar{\beta}_{1, i} F_{1, t}+\rho \bar{\beta}_{1, i+1} F_{1, t}+\rho \bar{\beta}_{2, i+1} F_{2, t}+\left.\sum_{j=2}^{\infty}\left(\rho^{j} W^{j} \bar{\beta} F_{t}\right)\right|_{i},
$$

where $\left.\right|_{i}$ identifies the $i$-th element of a vector. Note that equation (23) shows that, because of the network effect, asset $i$ increases the exposure to factor 1 of $\rho \bar{\beta}_{1, i+1}$ and is indirectly exposed to factor 2 of $\rho \bar{\beta}_{2, i+1}$, and the last term on the right represents further elements that can be specified only through the knowledge of the entire matrix $W$. Therefore, even if risky asset $i$ is not (structurally) exposed to a common factor (in the previous example, factor 2), the common factor will still play a role if it impacts on the returns of the assets to which $i$ is linked.

Such a result can be further generalized by focusing, for instance, on sector-specific risk factors. Those factors, in the presence of a network exposure, despite being sectorspecific, will have a systematic impact on all connected assets. Moreover, if we disregard the network exposure, we might also incur the risk of misinterpreting the impact of risk factors. In fact, by estimating the reduced-form model, we might label as common a factor that in reality is structurally related just to a subset of the investment universe and that impacts on other assets only through network connections.

A similar property exists for the idiosyncratic shocks. In fact, if we assume they are uncorrelated, the existence of network connections implies that the structural shocks of one asset impact on the returns of all the assets connected to it. Therefore, shocks on 
single assets can have effects on many other risky assets $9^{9}$ This evidence on the impact of a given asset's factor exposures and the shocks of linked assets resembles the decompositions typically adopted in spatial econometrics; see, among many others, LeSage and Pace (2009), as well as Ozdagli and Weber (2015) for a recent application. We note that the latter decompositions are appropriate in a framework where, for given dependent-variable measures across subjects, we have a number of covariates, each of which is available with variable-specific observations. In our case, we do have common factors (not assetspecific variables) and therefore these decompositions cannot be applied. From a pricing perspective, starting from the reduced-form representation we can easily show that the expected returns equal

$$
\mathbb{E}\left[R_{t}\right]=r_{f}+\bar{\beta} \Lambda+\sum_{j=1}^{\infty} \rho^{j} W^{j} \bar{\beta} \Lambda .
$$

Expected returns are thus influenced by network links that amplify the compensation for being exposed to the common factors. Further, we note that the pricing result depends on, and is thus conditional upon, the network structure, as summarized by $W$, which we assume to be known and time invariant. In fact, if we postulate that the coefficient $\rho$ is positive and that the elements of $W$ are all positive, the existence of links across assets induces higher expected returns than in the case of links being absent. Moreover, bearing in mind the previous discussion, the expected returns might depend on risk premiums associated with factors to which a given asset is not directly (structurally) exposed. The model estimation section discusses further elaborations on the risk premiums that emerge as consequences of the risk premium estimation.

In addition, we stress that the use of a network that is very dense, thus implying a matrix $W$ that is almost full, will have further impacts. In fact, a full $W$ implies that all idiosyncratic shocks are correlated. However, from our viewpoint, this corresponds to

\footnotetext{
${ }^{9}$ Summary measures of the exposure to common factors and idiosyncratic shocks can be obtained by mimicking the approaches used in spatial econometrics. A discussion on this topic is included in LeSage and Pace (2009); see their Section 2.7. These measures have been used in a financial framework by Asgharian, Less and Liu (2013). We also note that the decomposition of asset returns into four elements is equivalent to that proposed by Abreu et al. (2005) for separating the standard impact of covariates from the impact that is due to the spatial links, and is thus an alternative to the impact measures of LeSage and Pace (2009).
} 
indirect evidence of model misspecification as an additional common factor is now present but not taken into account. As a consequence, such common structural factor risk must be priced, and could generate the empirical evidence shown by Ang et al. (2006). The latter case could also correspond to empirical evidence challenging the validity of the APT approach. From a different viewpoint, our modelling framework still satisfies the assumptions required for APT. As we show in the next section, the presence of a network exposure, despite inducing correlation across the idiosyncratic shocks, does not exclude the existence of diversification benefits. In line with the network literature, we can also establish a link between our model and a measure of network centrality. In fact, Katz (1953) uses the expression in equation (18) to introduce his centrality measures. If we denote by $\mathbf{x}$ the score vector of the centrality, while $W$ is the adjacency matrix, $\mathbf{1}$ a vector of ones, and $\alpha$ and $C$ arbitrary constants, then Katz (1953) shows that the centrality vector equals

$$
\mathbf{x}=(I-\psi W)^{-1} \mathbf{1}
$$

with $\psi$ being a free parameter. Note that the $\psi$ manages the relationship between the centrality vector $\mathbf{x}$ and a limiting constant value for all centrality scores. In other words, if $\psi$ is zero all the nodes have the same centrality value. Otherwise, if $\psi$ assumes increasing values, $\sqrt{10}$ then $W$ plays an increasing role.

\subsection{Risk decomposition and portfolio diversification}

The model in equation (10) allows us to recover a risk decomposition similar to that available for the standard linear factor model in equation (2). The starting point is the reduced form introduced in equation (17). Equation (17) highlights that the estimation output of standard multifactor models can be consistent with the presence of contemporaneous links across assets, and this allows us to elaborate on the returns covariance structure.

\footnotetext{
${ }^{10}$ With an upper limit at $1 / \lambda_{\max }, \lambda_{\max }$ being the maximum eigenvalue of $W$, the value for which $(I-\psi W)^{-1}$ is non-singular.
} 
In fact, we can redefine $\mathcal{A}=(I-\rho W)^{-1}$, and then write the total variance of the risky assets as follows:

$$
\mathbb{V}\left[R_{t}\right]=\mathcal{A} \bar{\beta} \bar{\beta}^{\prime} \mathcal{A}^{\prime} \sigma_{m}^{2}+\mathcal{A} \Omega_{\eta} \mathcal{A}^{\prime}
$$

Despite being equivalent to the traditional risk decomposition of a multifactor model, equation (26) provides a relevant insight. In fact, both the systematic and idiosyncratic risk components are influenced by the presence of interconnections across assets, as the matrix $\mathcal{A}$ appears in both terms on the right hand side. This also shows that, if we estimate the reduced-form model using standard linear methods, our evaluations of the systematic and idiosyncratic risk components are in reality a blend between the structural loadings and idiosyncratic risks, and the network relations. Keiler and Eder (2013) suggest that the presence of spatial links could be interpreted as a systemic risk contribution. However, the previous decomposition provides an alternative view, in which spatial dependence is not an additive source of risk but rather a multiplicative one. In that case, the impact of the spatial dependence of a single asset cannot easily be recovered. In fact, the contribution to the asset risk due to the spatial dependence depends on both the structure of the network $W$ and the spatial parameter $\rho$. The two elements impact on the systematic contribution and on the idiosyncratic structural shock variances in a non-linear way. Obviously, the same structure appears at the portfolio level, where we have

$$
\mathbb{V}\left[r_{p, t}\right]=\omega^{\prime} \mathcal{A} \bar{\beta} \Sigma_{F} \bar{\beta}^{\prime} \mathcal{A}^{\prime} \omega+\omega^{\prime} \mathcal{A} \Omega_{\eta} \mathcal{A}^{\prime} \omega
$$

We first note that, if asset interconnections are not present (that is, when $\mathcal{A}=I$ ), the idiosyncratic risk equals $\Omega_{\eta}$ while the systematic risk component is $\bar{\beta} \Sigma_{F} \bar{\beta}^{\prime}$. We rewrite the portfolio variance decomposition in equation (27) by adding and subtracting the portfolio idiosyncratic and systematic variance components when they are not influenced by asset interconnections: 


$$
\mathbb{V}\left[r_{p, t}\right]=\omega^{\prime} \mathcal{A} \bar{\beta} \Sigma_{F} \bar{\beta}^{\prime} \mathcal{A}^{\prime} \omega+\omega^{\prime} \mathcal{A} \Omega_{\eta} \mathcal{A}^{\prime} \omega \pm \omega^{\prime} \bar{\beta} \Sigma_{F} \bar{\beta}^{\prime} \omega \pm \omega^{\prime} \Omega_{\eta} \omega
$$

With some rearrangement, the total portfolio variance can be recast into a decomposition comprising four different terms:

$$
\begin{aligned}
\mathbb{V}\left[r_{p, t}\right] & =\underbrace{\omega^{\prime} \bar{\beta} \Sigma_{F} \bar{\beta}^{\prime} \omega}_{I}+\underbrace{\left(\omega^{\prime} \mathcal{A} \bar{\beta} \Sigma_{F} \bar{\beta}^{\prime} \mathcal{A}^{\prime} \omega-\omega^{\prime} \bar{\beta} \Sigma_{F} \bar{\beta}^{\prime} \omega\right)}_{I I} \\
& +\underbrace{\omega^{\prime} \Omega_{\eta} \omega}_{I I I}+\underbrace{\left(\omega^{\prime} \mathcal{A} \Omega_{\eta} \mathcal{A}^{\prime} \omega-\omega^{\prime} \Omega_{\eta} \omega\right)}_{I V} .
\end{aligned}
$$

We make the following interpretations of the four risk components:

I the structural systematic risk component that depends on the structural loadings from the common factors and from the covariance of the common factors; this is the exogenous systematic effect;

II the effect of asset interconnections on the systematic risk component, or the first contribution of network exposure to the total risk; this is the endogenous systematic effect;

III the structural idiosyncratic component that depends only on the covariance of the structural shocks;

IV the effect of interconnections on the idiosyncratic risk, or the second contribution of network exposure to the total risk; this might be interpreted as an endogenous amplification of idiosyncratic risks.

Note that by adding the second and fourth terms we obtain the total contribution of network exposure to the total portfolio risk. We finally note that the model with asset interconnections is the standard multifactor model if there are no interconnections, that is if $W$ is a null matrix or if the coefficient $\rho$ is zero. 
In addition, the network exposure impacts on the idiosyncratic part of the variance. This implies that the diversification benefits might be endangered, depending on the network structure. In fact, despite the fact that the fourth term decreases with an increase in these cross-sectional dimensions, the speed of decrease is smaller than in the case without network effects.

Similarly to the standard linear factor model, we can recover analytical elements in a simplified setting. The covariance matrix $\Omega_{\eta}$ is diagonal; we further assume that the diagonal elements are set to an average value, $\bar{\sigma}^{2}=1$. In addition, we take an equally weighted portfolio and focus on the limiting case in which all assets are connected (thus $W$ has zeros only over the main diagonal, while off-diagonal terms equal $\frac{1}{K-1}$ after row normalization). In this case, we have that

$$
\begin{aligned}
\omega^{\prime} \mathcal{A} \Omega_{\eta} \mathcal{A}^{\prime} \omega & =\bar{\sigma}^{2} \omega^{\prime} \mathcal{A} \mathcal{A}^{\prime} \omega \\
& =\frac{\bar{\sigma}^{2}}{K^{2}} \mathbf{i}_{K}^{\prime} \mathcal{A} \mathcal{A}^{\prime} \mathbf{i}_{K} \\
& =\frac{K+\rho^{2}}{(K+\rho)^{2}(\rho-1)^{2}} \bar{\sigma}^{2}
\end{aligned}
$$

where $K$ is the number of assets and $\mathbf{i}_{K}$ is a $K$-dimensional vector of ones ${ }^{11}$ Moreover, we have that

$$
\lim _{K \rightarrow \infty} \frac{K+\rho^{2}}{(K+\rho)^{2}(\rho-1)^{2}} \bar{\sigma}^{2}=0,
$$

thus preserving the diversification benefit. However, the idiosyncratic risk contribution is higher than in the case without spatial dependence (i.e. with $\rho=0$ ). In fact, we can show that the above-reported portfolio idiosyncratic risk is higher than $\frac{1}{K} \bar{\sigma}^{2}$, thus confirming that term IV is positive.

\footnotetext{
${ }^{11}$ In the special case considered, the diagonal elements of $\mathcal{A}$ equal $\frac{(K-1) \rho-K}{\rho^{2}+(K-1) \rho-K}$ and the off-diagonal elements are $\frac{-\rho}{\rho^{2}+(K-1) \rho-K}$. Moreover, the diagonal elements of $\mathcal{A} \mathcal{A}$ equal $\frac{K \rho^{2}+[(K-1) \rho-K]^{2}}{\left[\rho^{2}+(K-1) \rho-K\right]^{2}}$ and the offdiagonal are $\frac{(K-1) \rho^{2}-2 \rho[(K-1) \rho-K]}{\left[\rho^{2}+(K-1) \rho-K\right]^{2}}$. Summing up the elements in $\mathcal{A} \mathcal{A}$ and simplifying, we obtain the above-reported result.
} 
The previous model thus gives a framework in which we can analyse the impact at the portfolio level of the interconnections we might observe across assets, and how those interconnections can endanger/limit the benefits of portfolio diversification.

\section{Model Generalizations}

In this section we provide two generalizations of the model by allowing asset-specific reaction to the network and introducing a time change into the network structure. We also add further discussion on the model's interpretation, in particular on the sign of the network-related parameters.

\subsection{Heterogeneous network reaction}

The model in equation (14) has, however, a very restricted structure. There is a single parameter, $\rho$, driving the network exposure. This can easily be generalized by allowing asset-specific responses to the network structure. We can thus modify the contemporaneous relation matrix of equation 15 into

$$
A=I-\mathcal{R} W
$$

where $\mathcal{R}=\operatorname{diag}\left(\rho_{1}, \rho_{2}, \ldots, \rho_{K}\right)$ is a diagonal matrix. This model is similar to the fixed coefficient specifications for spatial panels discussed in Elhorst (2003). A clear advantage of such a structure is given by the possibility that assets have different network exposures, as for each asset the model becomes

$$
R_{i, t}=\mathbb{E}\left[R_{i, t}\right]+\rho_{i} \sum_{j=1}^{k} w_{i, j}\left(R_{j, t}-\mathbb{E}\left[R_{j, t}\right]\right)+\bar{\beta}_{i} F_{t}+\eta_{i, t} .
$$

To estimate the asset-specific parameters, the network must satisfy an identification condition: each asset must be connected to at least one other asset. If this is not the case, 
the diagonal of matrix $\mathcal{R}$ must be restricted in such a way that unconnected assets will not have a network exposure. Further details will be discussed in the estimation section.

\subsection{On the sign of the coefficient $\rho$}

Up to this point, we have not discussed the sign of the coefficient $\rho$. Intuitively, we expect the assets to be positively related to one another, as the links come from a network. We thus imagine that shocks are transmitted to connected assets with their signs preserved. If we take a simplified model with a single coefficient $\rho$, it is highly improbable that we will ever observe negative coefficients. In fact, a single coefficient represents a sort of average reaction of the asset to the shocks coming from its neighbours.

However, in a model accounting for the heterogeneity of the reaction to the network exposure, negative asset-specific coefficients might appear. In other words, we cannot exclude a priori that a shock to one asset will lead to an opposite movement of a linked asset. We explain such a finding by making a parallel with negative correlations. If two assets are negatively correlated, their joint introduction to a portfolio will lead to a decrease of the overall variance. In a factor model, negative correlations across asset returns can be interpreted by loadings on the (same) common factors having different signs. In our framework, negative correlations across asset returns can emerge both in response to different signs in the factor loadings but also due to the presence of a negative asset-specific reaction to the network exposure.

Consider the reduced form of our model as represented in equation (30). In this case, the innovation term has a non-diagonal covariance. If we estimate the reduced-form model, the innovations could show evidence of non-null correlations, some of them being negative. They can be due both to the presence of opposite exposure to the common structural factors, whose coefficients have been estimated by a biased estimator (due to model misspecification), but also due to the presence of negative coefficients $\rho_{i}$.

In a general model with heteregeneous asset reactions to the network exposure, the components II and IV in the risk decomposition presented in equation (30) can become 
negative. In such a case, the network exposure reduces risk, and this could also be seen as a kind of flight-to-safety effect: if shocks hit financial assets and are then transmitted to industrial pro-cyclical sectors, we cannot exclude the possibility that the anti-cyclical sectors have an opposite network exposure.

Within our model, negative $\rho$ might thus exist, but how can we interpret them from a pricing perspective? We read them as evidence of risk absorption due to the network exposure. In fact, a negative $\rho_{i}$ allows a reduction of the exposure of one asset to the common factors, since the $i$-th component of the second term in equation (20) becomes negative. Risk absorption also has consequences for expected returns, leading to a reduction of the contribution of network exposure. In fact, the $i$-th component of the third term in equation (37) also becomes negative.

\subsection{Time change in the network structure}

The spatial econometrics literature generally assumes that the spatial proximity matrix is time invariant. In fact, if the matrix $W$ depends on physical measures, such as those is the spatial distance, they can safely be assumed to be constant over time. However, in a financial framework, the connections between assets might change over time for a number of reasons, such as, for instance, the occurrence of an unexpected market shock, or a merger or acquisition. Similar approaches have been adopted by Asgharian et al. (2013) and Keiler and Eder (2013). We are still assuming that the network is exogenous with respect to the linear structural model,${ }^{12}$ and the contemporaneous matrix can be further rewritten as

$$
A_{t}=I-\mathcal{R} W_{t}
$$

where we highlight that the network changes over time, and thus leads to a time-varying

\footnotetext{
${ }^{12}$ We might relax the exogeneity assumption by stating that the network is known conditional upon the past.
} 
matrix $W$. In turn, this induces time-dependence on the matrix $A$, as well as on the reduced-form parameter matrices, both on the betas and on the covariance of idiosyncratic shocks. That is, we also have heteroskedasticity. Nevertheless, we might postulate that the dynamic of $W_{t}$ is smooth, and operates at lower time scales than those monitoring the evolution of returns (for instance, we can assume the matrices $W$ change over the years, or after specific events such as crises). Therefore, the heteroskedasticity is mild, and the betas are evolving slowly. The use of time-varying matrices $W$ thus leads to a time change in the spatial dependence, differing from the approach of Blasques et al. (2014) who obtain the same result by letting the parameters $\mathcal{R}$ be time-varying. We note that, if the network exposure exists and the structural parameters in the matrix $\bar{\beta}$ are constant, the estimation of the reduced-form model over different samples might suggest changes in the factor exposure. However, those changes are not present but are due to the misspecification of the network relations. The expected returns are conditional upon the matrix $W$. If the network exposure is time-varying, the expected returns, conditional on $W_{t}$, are also time-varying.

A further issue associated with the change in $W_{t}$ over time is the normalization. In fact, if we let each single $W_{t}$ be row normalized, we could reduce the impact of changes in the network density: an increase in the number of assets linked to asset $j$ would lead to a decrease in the impact arising from a single asset, since the corresponding element of $W_{t}$ would diminish. As a consequence, with the introduction of the dynamic $W_{t}$ we also suggest considering a different normalization, which we refer to as a max row normalization. Formally, a non-normalized $W_{t}^{U}$ will be normalized as

$$
W_{i, j, t}=W_{i, j, t}^{U}\left(\max _{t} \sum_{i=1}^{N} W_{i, j, t}^{U}\right)^{-1}
$$

We stress that, when conditioning on the network structure, the pricing equation assumes the following form (in which we have also introduced asset-specific coefficients for network exposure): 


$$
\mathbb{E}\left[R_{t} \mid W_{t}\right]=r_{f}+\bar{\beta} \Lambda+\sum_{j=1}^{\infty}\left(\mathcal{R} W_{t}\right)^{j} \bar{\beta} \Lambda .
$$

The heterogeneity with respect to connections creates reactions to shocks on the common factors that differ across assets due to the different exposures of assets to the factors, but also due to the differing impacts of feedback loops coming from the underlying network structure. The change over time of the matrix $W_{t}$, or the presence of a structural break on the coefficients $\mathcal{R}$ (that we might find located close to the time of a crisis or extreme event), creates abrupt changes in the expected returns, and relevant movements in stock prices as a consequence. Therefore, the pricing, conditional upon the network structure, becomes a function of the network structure: if the network changes, the local equilibrium expected returns change. When we introduce a time variation into the matrices $W$, or into the elements $\mathcal{R}$, we must estimate risk premiums in the cross-sectional dimension but starting from the reduced-form model parameters and accounting for their time variation, as we will show in Section 5.1. We stress that, if we focus on a standard pricing model, we neglect the potential time variation in the expected returns.

\section{Model Estimation}

In the last section we formally introduced the theoretical framework. However, model parameters must be estimated and this poses various challenges. Let us consider the simultaneous model equation

$$
A R_{t}=\alpha+\bar{\beta} F_{t}+\eta_{t}
$$

Identification conditions are required to estimate the parameters of $A, \alpha, \bar{\beta}$ and the (diagonal) covariance matrix of $\eta_{t}$. The simple order condition of identification requires that the model parameters must be less than the parameters we can recover from the reduced-form specification. In fact, the latter can be estimated by least squares methods, 
and the structural parameters can be recovered thanks to their relation to the reducedform parameters. The reduced-form model is

$$
\begin{aligned}
R_{t} & =A^{-1} \alpha+A^{-1} \bar{\beta} F_{t}+A^{-1} \eta_{t} \\
& =\alpha^{\star}+\beta^{\star} F_{t}+\varepsilon_{t}^{\star},
\end{aligned}
$$

suggesting we can consistently estimate $K(M+1)$ mean parameters plus $\frac{1}{2} K(K+1)$ covariance parameters, where $M$ is the number of factors and $K$ the number of assets. However, an unrestricted structural specification, despite having the same number of parameters in the covariance, has $M K+K$ mean parameters.

The presence of asset interconnections, summarized into a network, allows a sensible reduction of the number of parameters included in the matrix $A$. In fact, if we have asset-specific network exposures and a single network, we have only $K$ parameters in $A$. However, this is not sufficient to provide identification of the model's remaining parameters, since the order condition is still not satisfied. Identification is obtained by imposing the diagonality of the covariance matrix of $\eta_{t}$. Such a choice, which is economically motivated, allows the standard order condition for identification to be satisfied.

Nevertheless, further constraints on the model parameters are generally required. Starting from the spatial econometrics literature, which takes a scalar time-invariant coefficient $\rho$ and a time-invariant row-normalized matrix $W$, we must impose $\frac{1}{\lambda_{\min }}<\rho<\frac{1}{\lambda_{\max }}$, where $\lambda_{\min }$ and $\lambda_{\max }$ are, respectively, the minimum and maximum eigenvalues of $W$. This constraint ensures the non-singularity of $A=I-\rho W$.

In our framework, we deviate from traditional approaches in several ways. We first consider the case of a time-varying spatial matrix, that is, $W_{t}$. A sufficient condition for the invertibility of $I-\rho W_{t}$ for all $t$ is stated in the following assumption: 
Assumption 5.1. The coefficient $\rho$ satisfies the following condition:

$$
\bar{\lambda}_{\min }^{-1}<\rho<\bar{\lambda}_{\max }^{-1}
$$

where

$$
\begin{aligned}
& \bar{\lambda}_{\text {max }}=\min \left\{\lambda_{t, \max }\right\}_{t=1}^{T} \\
& \bar{\lambda}_{\text {min }}=\max \left\{\lambda_{t, \min }\right\}_{t=1}^{T}
\end{aligned}
$$

and $\lambda_{t, \text { max }}$ and $\lambda_{t, \text { min }}$ are, respectively, the minimum and maximum eigenvalues of a matrix $W_{t}$.

If we have a diagonal matrix $\mathcal{R}$ containing the asset-specific reaction to the spatial links, we assume the non-singularity, which is then validated in the estimation step of the model:

Assumption 5.2. The diagonal coefficient matrix $\mathcal{R}$ is such that

$$
I-\mathcal{R} W_{t}
$$

is non-singular for each matrix $W_{t}$.

Note that the previous assumption covers both the case of a time-invariant and that of a time-varying spatial matrix. We further note that, when we consider a model with $\mathcal{R}$, we must impose an additional identification condition:

Assumption 5.3. The diagonal coefficient matrix $\mathcal{R}=\operatorname{diag}\left(\rho_{1}, \rho_{2}, \ldots, \rho_{K}\right)$ is such that $\rho_{j}=0$ if the matrix $W_{j}=\left[W_{j, 1}^{\prime} W_{j, 2}^{\prime} \ldots W_{j, T}^{\prime}\right]$, with $W_{j, t}$ being the $j$-th row of $W_{t}$, has non-null rank.

The previous assumption requires, irrespective of the number of matrices $W_{t}$, that if the $j$-th rows of all the matrices $W_{t}$ contain only zeros (that is, the asset $j$ is not linked to any other asset in the varying evolution of the network), then the asset $j$ 's network 
impact coefficient is restricted to zero as it cannot be identified. This condition ensures that the asset-specific impact on the network links is estimated only if such links exist for at least one point in time.

The use of covariance restrictions has a consequence for the estimation of the model parameters. In fact, they must be jointly evaluated, despite the fact that the linear model structure might allow for single-equation (single-asset) parameter estimation.

Under the two strong parametric restrictions we impose (the structure on $A$ and the absence of correlation across the idiosyncratic shocks), a viable approach is the Full Information Maximum Likelihood (FIML) method. However, if $K$ is even moderately large, the total number of parameters to be estimated in the restricted structural model, $M K$, might be quite large. Fortunately, we can follow the approaches commonly used in spatial econometrics, namely the use of concentrated likelihoods. As in Elhorst (2003), and LeSage and Pace (2009), we start by writing the full model's log-likelihood

$$
\begin{aligned}
L(\Theta) & =\sum_{j=1}^{T} l_{t}(\Theta), \\
l_{t}(\Theta) & \propto-\frac{1}{2} \log |\Omega|-\frac{1}{2} e_{t}^{\prime} \Omega^{-1} e_{t}, \\
e_{t} & =R_{t}-\bar{\alpha}-\mathcal{R} W R_{t}-\bar{\beta} F_{t},
\end{aligned}
$$

where $\Omega$ is a diagonal matrix. If the parameters in $\mathcal{R}$ are known, we can write

$$
R_{t}-\mathcal{R} W R_{t}=Z_{t}=\bar{\alpha}+\bar{\beta} F_{t}+\varepsilon_{t} .
$$

Therefore, with a known network exposure parameter matrix $\mathcal{R}$, with a unique (and even time-variant) network structure, we can estimate the parameters in $\bar{\alpha}$ and in $\bar{\beta}$ by least squares methods, obtaining the well-known expressions. In addition, we can even recover standard estimators for the innovation variance. This suggests that the network exposure parameters can easily be obtained by maximizing the concentrated likelihood 
obtained by replacing the other parameters with their least squares estimators. ${ }^{13}$

\subsection{Risk premium estimation}

We now tackle the crucial issue of risk premium estimation in our framework. The literature generally follows two approaches, the Fama and French (1993) or the Fama and McBeth (1973) method. Similarly to Ahern (2013), for the network factors, we follow the Fama and McBeth (1973) two-pass method. Therefore, we plan to estimate the risk premiums starting from the time-series estimate of the betas and then taking a cross-sectional regression.

To highlight the links existing between the traditional multifactor model and our proposal, we first rewrite the two models, the linear factor model and the factor model augmented with network-driven contemporaneous relations, respectively, reporting the second in its reduced-form representation:

$$
\begin{aligned}
& R_{t}=\alpha+\beta F_{t}+\varepsilon_{t} \\
& R_{t}=\alpha^{\star}+\beta^{\star} F_{t}+\varepsilon_{t}^{\star} .
\end{aligned}
$$

Under the traditional factor model, in which the $M$ factors do have a zero mean, the Fama-McBeth procedure corresponds to a collection of $K$ time-series regressions of the form

$$
R_{i, t}=\alpha_{i}+\beta_{i} F_{t}+\varepsilon_{i, t}
$$

followed by a cross-sectional regression

\footnotetext{
${ }^{13}$ This is of relevant computational importance as it allows us to reduce the parameters to be jointly estimated to $2 K$ if we concentrate the likelihood with respect to $\bar{\alpha}$ and $\bar{\beta}$, and to $K$ if we also concentrate with respect to the innovation variance. Standard errors can be recovered from the full-model likelihood by making numerical evaluations of the Hessian (and of the gradient if we take a robust-parameters covariance matrix). Note that this approach can be followed even if the spatial matrix $W$ is time-varying, or with zero restrictions added to specific parameters of $\mathcal{R}$ to ensure model identification.
} 


$$
R^{e}=\hat{\beta} \Lambda+\nu,
$$

where $R^{e}$ is the $K$-dimensional vector of the sample averages of excess returns, $\hat{\beta}$ is the $K$-by- $M$ matrix of (least squares) factor loadings estimates and $\Lambda$ is the unknown vector of risk premiums. The estimates suffer from the error-in-variable issue. In the empirical part we manage the error-in-variable issue by working on industry portfolios. Further, we introduce the Shanken (1992) correction into the estimation of the standard errors.

We start by focusing on the case with heterogeneous network impact and time-invariant $W$. In that case, the risk premiums and the expected asset returns satisfy the following equilibrium condition:

$$
\mathbb{E}\left[R_{t}\right]=r_{f}+\bar{\beta} \Lambda+\sum_{j=1}^{\infty} \mathcal{R}^{j} W^{j} \bar{\beta} \Lambda=r_{f}+\beta^{\star} \Lambda .
$$

Therefore, in our model, the estimation of the risk premium using the Fama-McBeth approach points at using the reduced-form factor loadings $\beta^{\star}$. Consequently, we first estimate the model and then run the cross-sectional regression

$$
\bar{R}^{e}=\widehat{\beta^{\star}} \Lambda+\nu^{\star},
$$

where we have replaced the reduced-form betas with their estimates. By simple comparison of the two second-stage (cross-sectional) regressions, we can state the following proposition:

Proposition 5.1. If the true model includes a contemporaneous relation driven by a timeinvariant network with heterogeneous asset impact, the Fama-McBeth approach based on ordinary least squares (OLS) leads to a consistent estimation of the risk premiums even if we estimate the factor loadings using the misspecified traditional factor model. In other words, the OLS estimates of $\Lambda$ based on $\hat{\beta}$ and $\hat{\beta}^{\star}$ do coincide.

Proof. This is a consequence of the fact that, if $W$ is time-invariant, the least squares 
estimator applied to the model in equation (50) provides a consistent estimate of our model's reduced-form betas, $\beta^{\star}$. In other words, $\beta \equiv \beta^{\star}$, and thus the risk premiums also coincide.

Few remarks are then needed. First, the previous result holds in the special case of scalar network impact. Second, the result holds only for the point estimates of the risk premiums and only if we estimate the premiums by standard least squares. This is due to the fact that, under our model, the covariance of $\varepsilon_{t}^{\star}$ has a known structure that depends on the network, i.e. the matrix $W$, and on the parameters included in $\mathcal{R}$. This covariance differs from the estimated covariance of $\varepsilon_{t}$ under the multifactor model. Consequently, inference provides, potentially, different outcomes, that is different standard errors. Furthermore, if we adopt generalized least squares, even the (small-sample) point estimates differ.

The previous proposition allows us to highlight that, when the network is timeinvariant, the risk premium estimation is unaffected by the model misspecification. Consequently, our model's main contributions are as follows: (i) the ability to disentangle the structural and the network-induced factor exposure; (ii) the possibility of capturing correlation in the residuals of traditional linear factor models; (iii) the possibility of showing that diversification benefits are weaker than expected.

When the network is dynamic, the expected returns are characterized by

$$
\mathbb{E}\left[R_{t} \mid W_{t}\right]=r_{f}+\bar{\beta} \Lambda+\sum_{j=1}^{\infty} \mathcal{R}^{j} W_{t}^{j} \bar{\beta} \Lambda=r_{f}+\beta_{t}^{\star} \Lambda .
$$

We first point out that the reduced-form betas are time-varying, being equal to $\beta_{t}^{\star}=$ $\left.\left(1-R W_{t}\right)^{(}-1\right) \bar{\beta}$, while we have time-invariant risk premiums.

From the previous expected return relation we have that, under our model with timevarying networks, the Fama-McBeth approach for risk-premium estimation involves a second-stage cross-sectional regression in which we still focus on reduced-form betas but must account for the fact that those betas are time-varying. 
Let us assume that the network changes at a time frequency much lower than that for the returns data we use for model and risk factor exposure estimation. We thus have a sample of $T$ observations for returns and $N<<T$ networks. Further, let us assume that the networks change every $T / N=m$ observations. First, we must define sample averages of excess returns as follows:

$$
R_{i}^{e}=\frac{1}{T / N} \sum_{t=\frac{T}{N}(i-1)+1}^{t=\frac{T}{N} i}\left(R_{t}-r_{f, t}\right), \quad i=1,2, \ldots N
$$

Then, the cross-sectional regression becomes

$$
\left[\begin{array}{c}
R_{1}^{e} \\
R_{2}^{e} \\
\vdots \\
R_{N}^{e}
\end{array}\right]=\left[\begin{array}{c}
\beta_{1}^{\star} \\
\beta_{2}^{\star} \\
\vdots \\
\beta_{N}^{\star}
\end{array}\right] \Delta+\nu
$$

where the time-varying reduced-form betas take exactly $N$ different values.

Two relevant remarks originate from the modified Fama-McBeth procedure, we must adopt within our model.

Remark 1. If we adopt a misspecified linear factor model, we do not capture the time change in the betas. This rationalizes, at least in part, the observed time change in the factor exposures. Moreover, the estimation of risk premiums might differ between the two models, i.e. the multifactor model and our proposal augmented with network exposure. Let us assume the following:

A1 the network does not collapse on absorbing states with the absence of connections or the presence of full connections,

A2 the average returns converge to a long-run value $\frac{1}{N} \sum_{i=1}^{N} R_{i}^{e} \rightarrow_{p} R^{e}$,

A3 the reduced-form betas converge to a long-run value $\frac{1}{N} \sum_{i=1}^{N} \beta_{i}^{\star} \rightarrow_{p} \beta^{\star}$,

A4 the risk premiums are time-invariant, 
where $\rightarrow_{p}$ denotes convergence in probability.

Under the previous assumptions, the point estimation of the risk premiums under the classical linear factor model is, again, not affected by the introduction into the model of the network exposure, and Proposition 5.1 remains valid in the limit. This is a consequence of the time-invariance of the risk premiums.

Remark 2. The availability of $N$ different cross-sectional collections of reduced-form betas also allows us to devise a strategy for testing the stability of the risk premiums. In fact, assuming the correct model specification, we have an estimate of the risk premiums from the $N$ regressions of the form

$$
R_{i}^{e}=\beta_{i}^{\star} \Delta+\nu_{i}, \quad i=1,2, \ldots N
$$

where the risk premiums are assumed to be identical. Therefore, testing the equality of the risk premiums across the $N$ estimates corresponds to a test for the stability of the risk premiums, under the assumption of stability of the structural factor exposures. This is feasible if we redefine the $N$ equations in (57) in a seemingly unrelated regression (SURE) model. In that case, the full model becomes

$$
\left[\begin{array}{c}
R_{1}^{e} \\
R_{2}^{e} \\
\vdots \\
R_{N}^{e}
\end{array}\right]=\left[\begin{array}{cccc}
\beta_{1}^{\star} & 0 & \ldots & 0 \\
0 & \beta_{2}^{\star} & \ldots & 0 \\
\vdots & \vdots & \ddots & \vdots \\
0 & 0 & \ldots & \beta_{N}^{\star}
\end{array}\right]\left[\begin{array}{c}
\Delta_{1} \\
\Delta_{2} \\
\vdots \\
\Delta_{N}
\end{array}\right]+\nu,
$$

and it is thus natural to test for the validity of the null hypothesis of equality across the $N$ risk premiums 14

\footnotetext{
${ }^{14}$ Obviously, the SURE model allows for several intermediate specification lying between the case of a single vector of risk premiums over time and the existence of $N$ different risk premium vectors. Those various cases can easily be compared to the limiting ones using standard approaches.
} 


\section{Simulation Analysis}

To show the capabilities of the proposed framework and to underline the effect due to model misspecification, that is, neglecting the network links across assets, we include in this section a set of simulations.

\subsection{Scalar network impact}

First, we concentrate on the simplest model, with time-invariant $W$ and scalar $\rho$. Such a baseline design will provide some expected results, as we will point out in a few lines. The first data-generating process we consider is a linear factor model with a unique risk factor, a scalar network impact and a fixed (and known a priori) network matrix $W$ :

$$
(I-\rho W)\left(R_{t}-\mathbb{E}\left[R_{t}\right]\right)=\bar{\beta} F_{t}+\eta_{t},
$$

with the following specification for parameters, shocks and asset interconnections:

- We consider $K=100$ assets, thus focusing on a somewhat large cross-sectional dimension, and assume we simulate monthly returns.

- The coefficient $\rho$ assumes fixed values $\rho \in\{0,0.25,0.5,0.75\}$, allowing us to compare the case of no network impact with different and increasing levels of network impact; note that, when $\rho=0$, our model collapses to the traditional linear factor model.

- The factor loading coefficients are randomly generated from $\beta_{i} \sim \mathcal{U}(0.8,1.2), i=$ $1,2, \ldots, K$, giving positive factor loadings with an average value of 1 .

- We simulate the factor returns from a Gaussian density, $F_{t} \sim \mathcal{N}\left(\mu_{F}, \sigma_{F}^{2}\right)$ with $\mu_{F}=0$ and $\sigma_{F}=15 \%$, on a yearly basis.

- The risky asset's expected return equals $\mathbb{E}\left[R_{t}\right]=r_{f}+(I-\rho W)^{-1} \beta \Lambda, \beta$ being the $K$-dimensional vector of betas simulated above, while the factor risk premium equals $5 \%$ on a yearly basis, and the risk-free rate is set to $1 \%$ on a yearly basis. 


\begin{tabular}{lcccccccc}
\hline$T$ & \multicolumn{2}{c}{$\rho=0$} & \multicolumn{2}{c}{$\rho=0.25$} & \multicolumn{2}{c}{$\rho=0.5$} & \multicolumn{2}{c}{$\rho=0.75$} \\
& Mean & Std.dev & Mean & Std.dev & Mean & Std.dev & Mean & Std.dev \\
\hline \multicolumn{8}{c}{ Distortions for $\rho$} \\
\hline 200 & 0.067 & 0.084 & 0.062 & 0.075 & 0.050 & 0.058 & 0.028 & 0.032 \\
500 & 0.029 & 0.040 & 0.028 & 0.037 & 0.024 & 0.030 & 0.014 & 0.017 \\
1000 & 0.015 & 0.024 & 0.014 & 0.023 & 0.012 & 0.019 & 0.007 & 0.011 \\
\hline \multicolumn{8}{c}{ Cross-sectional average of the distortions $\beta$} \\
\hline 200 & -0.072 & 0.091 & -0.088 & 0.107 & -0.096 & 0.107 & -0.119 & 0.133 \\
500 & -0.032 & 0.043 & -0.040 & 0.053 & -0.045 & 0.061 & -0.059 & 0.072 \\
1000 & -0.016 & 0.026 & -0.020 & 0.032 & -0.022 & 0.034 & -0.031 & 0.045 \\
\hline
\end{tabular}

Table 1: Mean and standard deviation for the distortions $\rho$ and $\beta$ under a correct model specification across different values of the network impact and different sample sizes. Values computed across 500 replications.

- The matrix $W$ comes from a simple and naive design: each of its off-diagonal elements is extracted from a Bernoulli density $w_{i, j} \sim \mathcal{B}\left(p_{B}\right)$ with $p_{B}=0.3$; the simulated $W$ is then row-normalized.

- The shocks are extracted from a Gaussian $\eta_{t} \sim \mathcal{N}(0, \Omega)$, with $\Omega$ being a diagonal matrix with diagonal elements extracted from a uniform, $\omega_{i, i}^{\frac{1}{2}} \sim \mathcal{U}(10 \%, 25 \%)$ with limits referring to a yearly horizon.

- We simulate 500 sequences of monthly returns with three different sample sizes, $T=200,500$ and 1000 .

The baseline simulation provides expected results. Firstly, the estimators of the coefficients $\rho$ and of the (structural) vector $\beta$ have an asymptotically normal density with dispersion decreasing with the sample size (see Table11). Figure1 1 reports a kernel estimate of the distortion $\hat{\rho}-\rho$ across different values of $\rho$, while Figure 2 provides a kernel density for the cross-sectional average (over the assets) of the distortions $\hat{\beta}_{i}-\beta_{i}, i=1,2, \ldots, K$; all graphs show the plots for the three different sample sizes. We note that the coefficients converge to the true values and that their dispersion decreases with the sample size, as expected.

If we estimate a standard linear factor model on the series simulated from equation (59), that is, we fit 


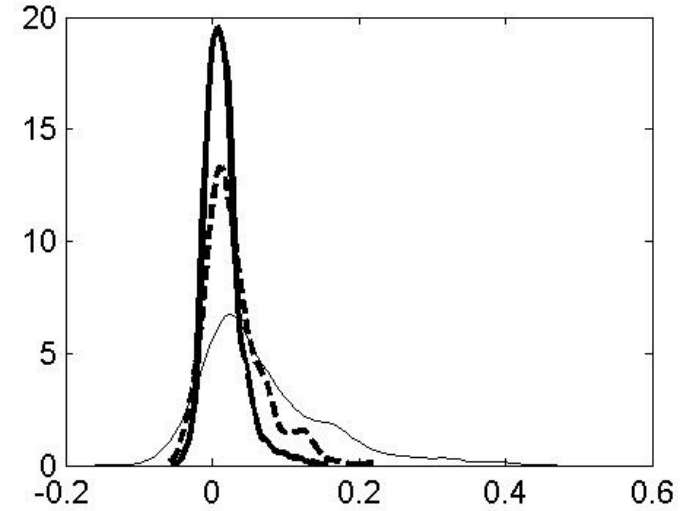

(a)

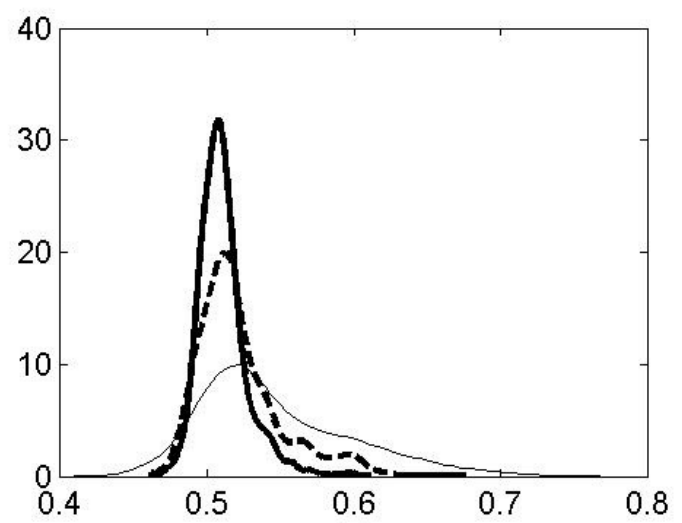

(c)

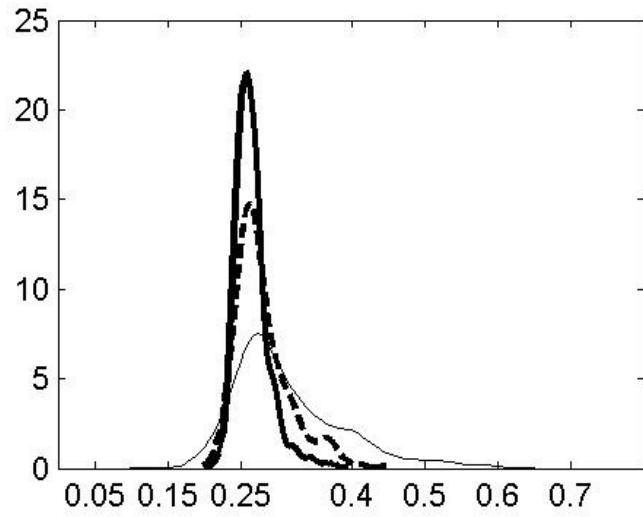

(b)

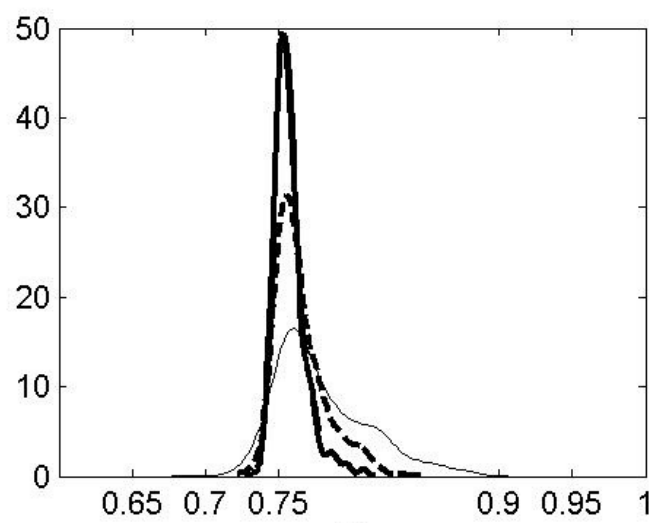

(d)

Figure 1: Distortions of the coefficients $\rho$ under the correctly specified model. True values: (a) $\rho=0$, (b) $\rho=0.25$, (c) $\rho=0.5$ and (d) $\rho=0.75$. Lines refer to different sample sizes, $T=200$ thin grey line, $T=500$ dashed line and $T=1000$ thick black line. 


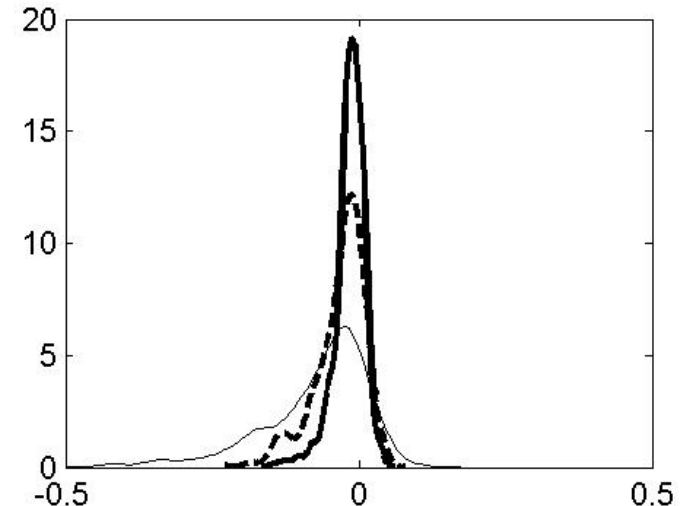

(a)

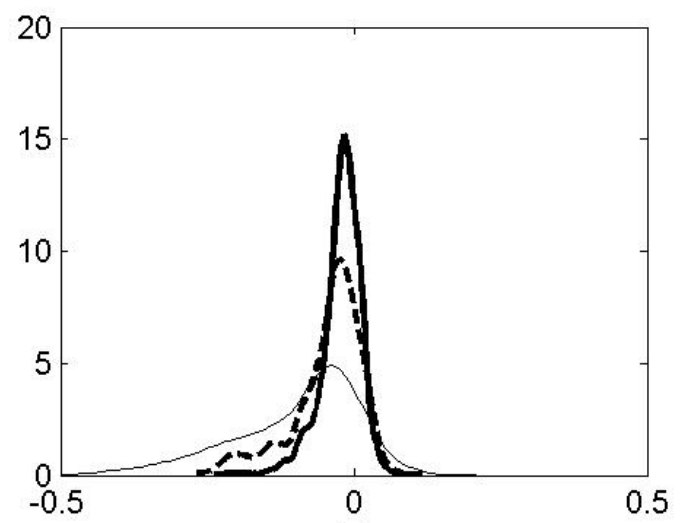

(c)

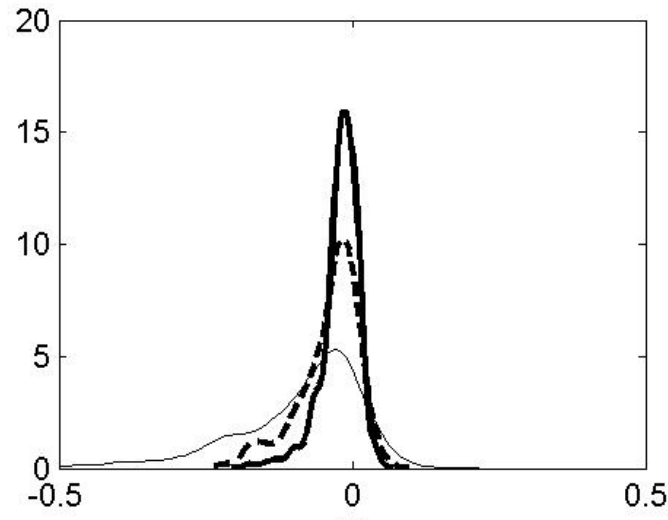

(b)

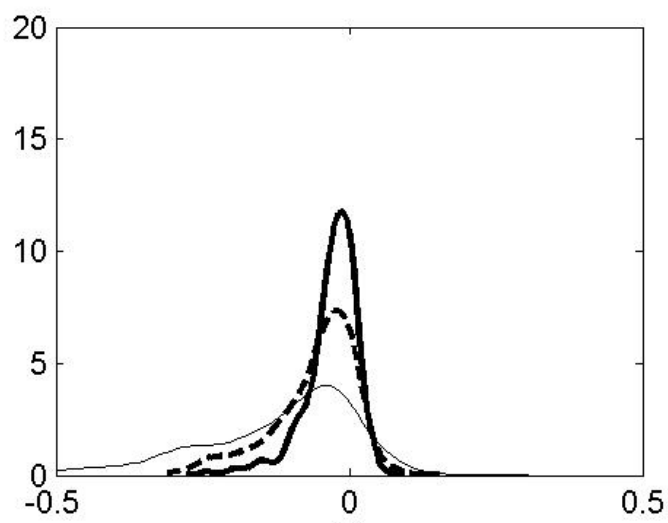

(d)

Figure 2: Cross-sectional average of the distortions for the coefficients $\beta$ under the correctly specified model across different values of $\rho$. True values: (a) $\rho=0$, (b) $\rho=0.25$, (c) $\rho=0.5$ and (d) $\rho=0.75$. Lines refer to different sample sizes, $T=200$ thin grey line, $T=500$ dashed line and $T=1000$ thick black line. 


\begin{tabular}{|c|c|c|c|c|c|c|c|c|}
\hline \multirow[t]{2}{*}{$T$} & \multicolumn{2}{|c|}{$\rho=0$} & \multicolumn{2}{|c|}{$\rho=0.25$} & \multicolumn{2}{|c|}{$\rho=0.5$} & \multicolumn{2}{|c|}{$\rho=0.75$} \\
\hline & lean & Std.dev & Mean & Std.dev & Mean & Std.dev & Mean & Std.dev \\
\hline & \multicolumn{8}{|c|}{ Cross-sectional average of the distortions $\hat{\gamma_{1}}-\beta$} \\
\hline 200 & 00 & 0.026 & 0.337 & 0.027 & 1.013 & 0.028 & 3.033 & 0 \\
\hline 500 & & & & & & & & \\
\hline \multirow[t]{2}{*}{1000} & 0.000 & 0.011 & 0.337 & 0.012 & 1.012 & 0.012 & 3.035 & 0.019 \\
\hline & \multicolumn{8}{|c|}{ Average residual correlations under the misspecified linear factor model } \\
\hline 200 & 0.000 & 0.003 & 0.009 & 0.0 & 0.034 & 0.014 & 0.149 & 0.035 \\
\hline 500 & & ( & 0 & & 0.0 & & 0 . & 35 \\
\hline 1000 & 000 & 0.001 & 0.009 & 0.006 & 0.033 & 0.013 & 0.149 & 0.035 \\
\hline \multicolumn{9}{|c|}{ Average residual correlations under the correctly specified model } \\
\hline 200 & 001 & 0.004 & -0.002 & & -0.002 & & -0.002 & 0.003 \\
\hline 500 & 001 & 002 & -0.0 & 0.0 & -0.001 & 0. & -0.001 & 0.002 \\
\hline 1000 & 0.000 & 0.001 & 0.000 & 0.001 & 0.000 & 0.001 & -0.001 & 0.001 \\
\hline
\end{tabular}

Table 2: Mean and standard deviation for the cross-sectional average of the distortions $\hat{\gamma}_{1}-\beta$ under model misspecification, upper panel; average residual correlation under model misspecification, central panel, and under correct model specification, lower panel. Statistics computed across different values of the network impact and different sample sizes. Values computed across 500 replications.

$$
R_{t}=\gamma_{0}+\gamma_{1} F_{t}+\varepsilon_{t}
$$

we have that $\gamma_{0}=\mathbb{E}\left[R_{t}\right], \gamma_{1}=(I-\rho W) \beta$, and $\mathbb{V}\left[\varepsilon_{t}\right]=(I-\rho W)^{-1} \Omega\left(I-\rho W^{\prime}\right)^{-1}$. Therefore, estimating the linear factor model we estimate the reduced-form representation of our model with network dependence. The $\gamma_{1}$ coefficients, by construction, will be larger than the structural coefficients $\beta$ when we simulate from a data-generating process with positive $\rho$. This is confirmed by Figure 3 and Table 2 in which we report the kernel density for the cross-sectional average of $\hat{\gamma}_{1, i}-\beta_{i}, i=1,2, \ldots K$ and some descriptive statistics. Moreover, the residuals of the linear factor model will be correlated, with average correlation increasing with $\rho$ (see Table 2).

Figure 3 and Table 2 confirm that, by fitting a linear factor model, we estimate a beta much larger than the structural value, with distortion increasing with the impact from the network connections. As a consequence, the value of the true and structural factor loading might sensibly differ from the one that is empirically observed, being doubled for 


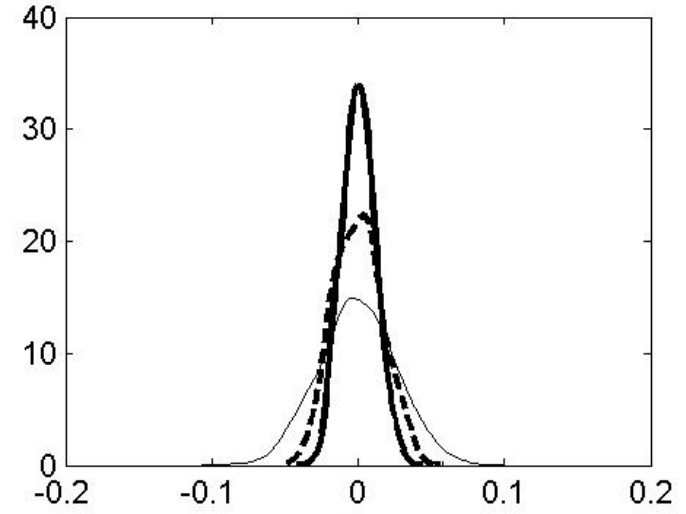

(a)

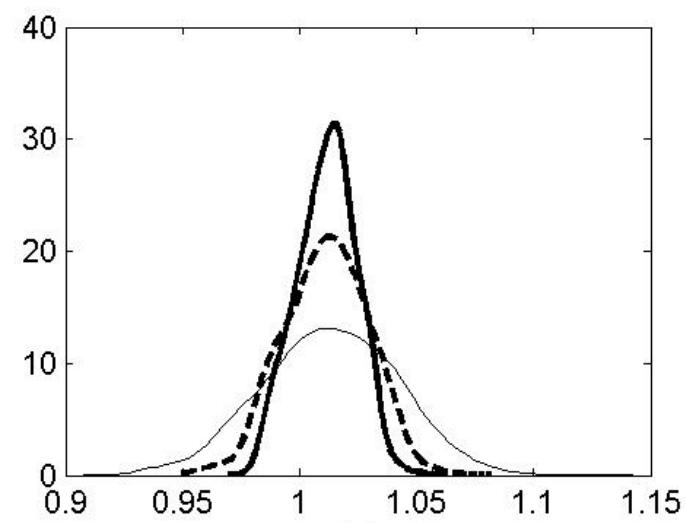

(c)

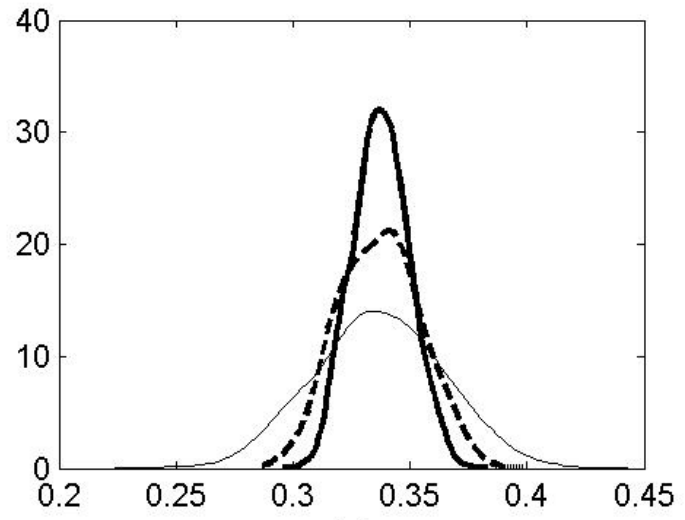

(b)

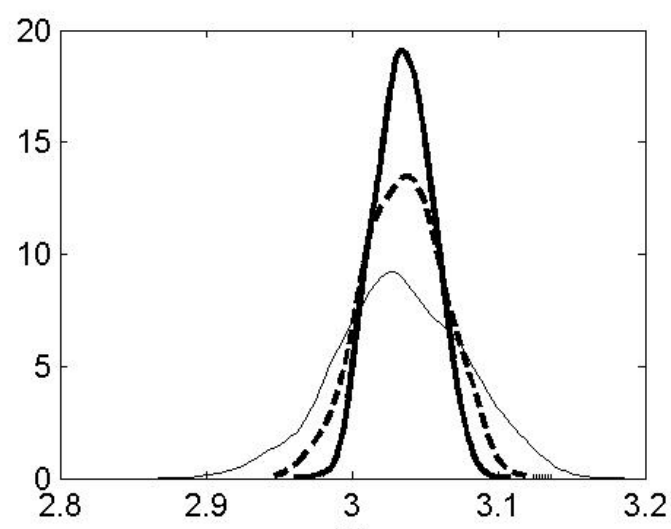

(d)

Figure 3: Cross-sectional average of the distortions $\hat{\gamma}_{1}-\beta$ under the misspecified model across different values of $\rho$ for the data-generating process. True values: (a) $\rho=0$, (b) $\rho=0.25$, (c) $\rho=0.5$ and (d) $\rho=0.75$. Lines refer to different sample sizes, $T=200$ thin grey line, $T=500$ dashed line and $T=1000$ thick black line. 
values of $\rho$ equal to 0.5 , and thus not particularly elevated.

When analysing the residual correlations, we can see that they are zero when the linear factor model is correctly specified, that is, when $\rho=0$. However, in the presence of a network impact, the residual correlations start drifting away from zero, their values increasing with $\rho$. On the contrary, under the correct model specification, the residual correlations are almost zero, as expected.

Next, we move to the estimation of the factor risk premium. We adopt the widely used two-pass regression approach of Black et al. (1972) and Fama and McBeth (1973). In linear factor models the first stage corresponds to the estimation of the factor loadings, that is, the betas. Differently, in our model the first stage is the estimation of reducedform betas starting from the estimated coefficient $\rho$ and corresponds to a by-product of the concentrated maximum likelihood estimation approach adopted. We stress that, under scalar $\rho$ and with a static $W$, the reduced-form betas and the linear factor model betas are asymptotically equivalent. The second regression is a cross-sectional one, which takes as dependent the average risky asset returns and regresses them on the estimated betas (reduced-form betas in our model). As pointed out by Black et al. (1972), the estimated risk premium suffers from an error-in-variable problem and is thus inconsistent. Standard solutions include grouping assets into portfolios, increasing the sample size and increasing the cross-sectional dimension. We apply the second one, since we are working in a purely simulated setting in which we do not control for the risky asset's market value. As a consequence, we expect distortions in the estimation of the risk premiums for small sample sizes, and, given the asymptotic equivalence of the betas, no difference between our model and the misspecified linear factor model. However, an expected result like this does not impact on the purpose of our simulation design, as our final objective is not the correct estimation of the risk premiums but rather to highlight the differences in the estimated risk premiums obtained from either a correctly specified model or a misspecified linear factor model. We finally point out that the cross-sectional estimation of the risk premium could come from either a standard OLS or a GLS estimator. For the latter, we 


\begin{tabular}{lcccccccc}
\hline$T$ & \multicolumn{2}{c}{$\rho=0$} & \multicolumn{2}{c}{$\rho=0.25$} & \multicolumn{2}{c}{$\rho=0.5$} & \multicolumn{2}{c}{$\rho=0.75$} \\
& Mean & Std.dev & Mean & Std.dev & Mean & Std.dev & Mean & Std.dev \\
\hline \multicolumn{8}{c}{ Estimated risk premiums from a linear factor model } \\
\hline 200 & 0.415 & 0.317 & 0.417 & 0.318 & 0.418 & 0.318 & 0.419 & 0.319 \\
500 & 0.417 & 0.198 & 0.418 & 0.199 & 0.418 & 0.199 & 0.418 & 0.199 \\
1000 & 0.423 & 0.137 & 0.423 & 0.138 & 0.423 & 0.138 & 0.423 & 0.138 \\
\hline \multicolumn{8}{c}{ Estimated risk premiums from a correctly specified model } \\
\hline 200 & 0.418 & 0.322 & 0.419 & 0.323 & 0.420 & 0.324 & 0.421 & 0.325 \\
500 & 0.418 & 0.200 & 0.419 & 0.200 & 0.419 & 0.200 & 0.419 & 0.200 \\
1000 & 0.423 & 0.138 & 0.423 & 0.138 & 0.424 & 0.138 & 0.424 & 0.138 \\
\hline
\end{tabular}

Table 3: Mean and standard deviation of the estimated risk premiums across the 500 replications. The cross-sectional regression adopts an OLS estimator. The true risk premium corresponds to 0.4167 at the monthly frequency.

note that the correct model specification allows for a more precise design of the residuals' covariance (in the reduced-form representation of our model).

In Table 3 we report the estimated risk premiums. As expected, the premiums are very close to the true value, with a dispersion decreasing in $T$. The limited distortions depend on the large sample sizes we consider ${ }^{15}$ No difference emerges when we compare the correctly and incorrectly specified models. Finally, we should point out that the OLS and GLS estimators provide substantially equivalent results, and thus we report only the OLS case.

As a further example, we consider the portfolio variance, $1 / N$, concentrating on the role played by the idiosyncratic risks. We order assets on the basis of their idiosyncratic risk and decompose the portfolio idiosyncratic risk into the structural component and the network effect. We consider portfolios with $N$ varying from 5 to 100 . Figure 4 reports the decomposition in both absolute and relative terms. Notably, network exposure induces a decrease in the idiosyncratic risks that is much smaller than that associated solely with the structural risks, and with a relative weight increasing over time. Such a result leads to diversification benefits that are reduced compared to the ideal case of independent idiosyncratic shocks (associated with the reduced-form model representation).

To evaluate the impact of the various settings of the data-generating process, we run

\footnotetext{
${ }^{15}$ Similar results have been obtained with smaller samples of 60 and 120 observations.
} 

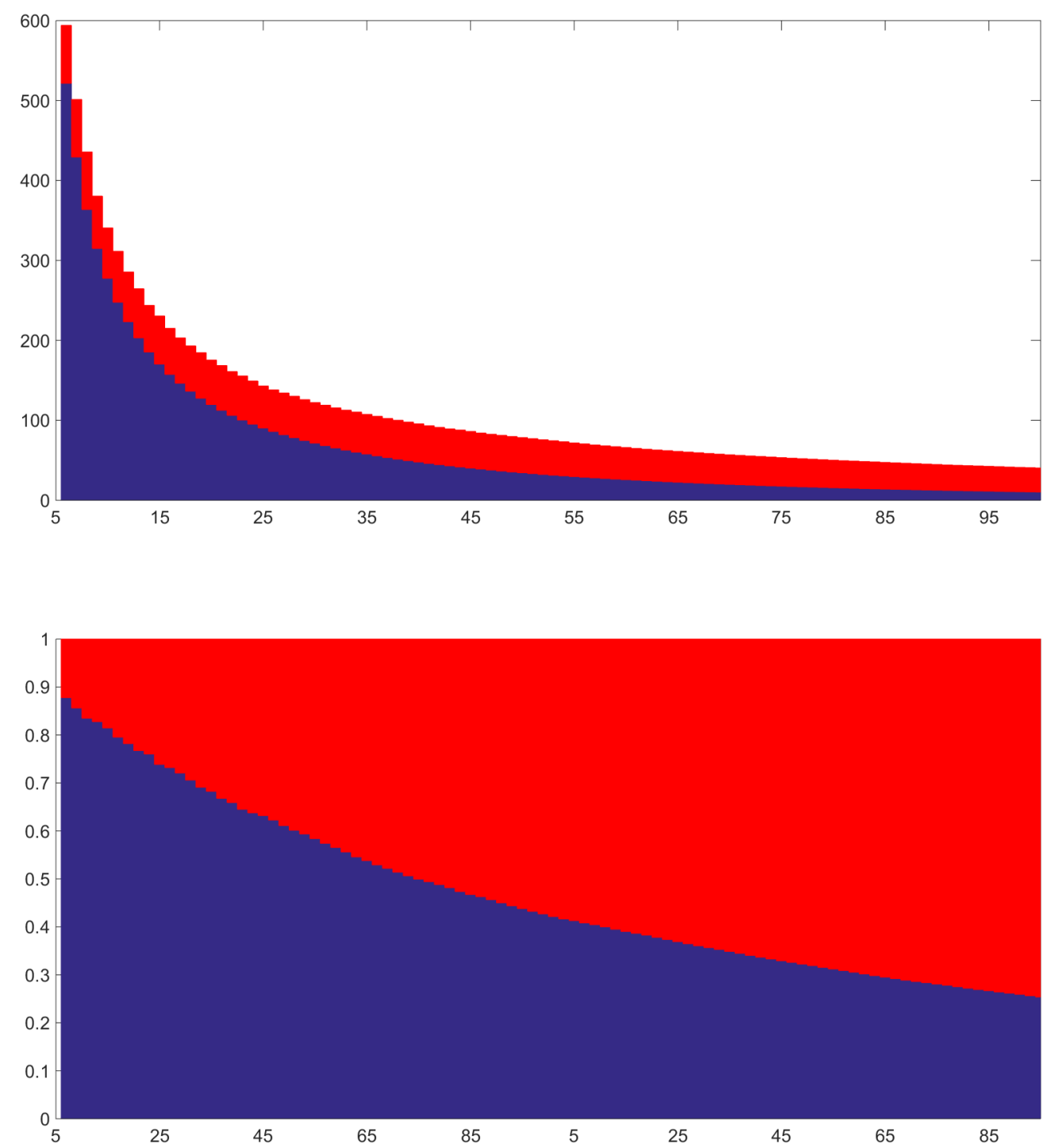

Figure 4: $1 / N$ portfolio idiosyncratic risk components: structural risk (blue) and networkrelated risk (red) across different portfolio sizes using the same assets adopted in the simulations and with $\rho=0.5$. Absolute decomposition (upper) and relative decomposition (lower). 
a number of robustness checks: we simulate the vector $\beta$ from a Gaussian with mean 1 so that the betas are more concentrated around the mean but also characterized by a larger variance; we increase the volatility of the common factor to a yearly value of $25 \%$; we change the network density by setting $p_{B}=0.15$ and $p_{B}=0.45$, or, maintaining the same density, we simulate different networks; we modify the factor risk premium to $\Lambda=3 \%$ or $\Lambda=10 \%$; we increase the relevance of the idiosyncratic shocks by sampling elements of $\Omega$ as $\omega_{i, i}^{\frac{1}{2}} \sim \mathcal{U}(20 \%, 50 \%)$.

None of these changes affects the previously reported results ${ }^{16}$

\subsection{Heterogeneous network impact}

The second simulation design we consider adds the heterogeneity to the network impact. We thus move from the coefficient $\rho$ to the diagonal matrix $\mathcal{R}$. The asset-specific network impact comes from a normal density, $\rho_{i} \sim \mathcal{N}(0.5,0.01)$, such that with probability close to $99 \% \rho$ takes values between 0.25 and 0.75 . In order to control the computational time, we reduce the cross-sectional dimension for this simulation and set $K=20$.

For that case, we provide in Figure 5 a kernel density for the cross-sectional average of $\hat{\rho}_{i}-\rho_{i}, i=1,2, \ldots, K$ for different sample sizes.

We do not present further results for the estimated factor loadings and residual correlations associated with the fit of the standard linear factor model as they provide the same evidence as in the first simulation design: the betas are larger than the structural values and the residuals are correlated. We only point out that, in the presence of heterogeneity in the network impact, the residual correlations are even higher than in the case of scalar $\rho{ }^{17}$

Instead, we provide in Table 4 further evidence from the risk premium estimation. Notably, the estimated risk premiums present a slight distortion (overestimation) in relation to the previous simulation design. We link them to the introduction of the assetheterogeneous impact of the network, which intuitively amplifies the impact of the error-

\footnotetext{
${ }^{16}$ Additional figures and tables are available upon request.

${ }^{17}$ Additional tables and figures are available upon request.
} 


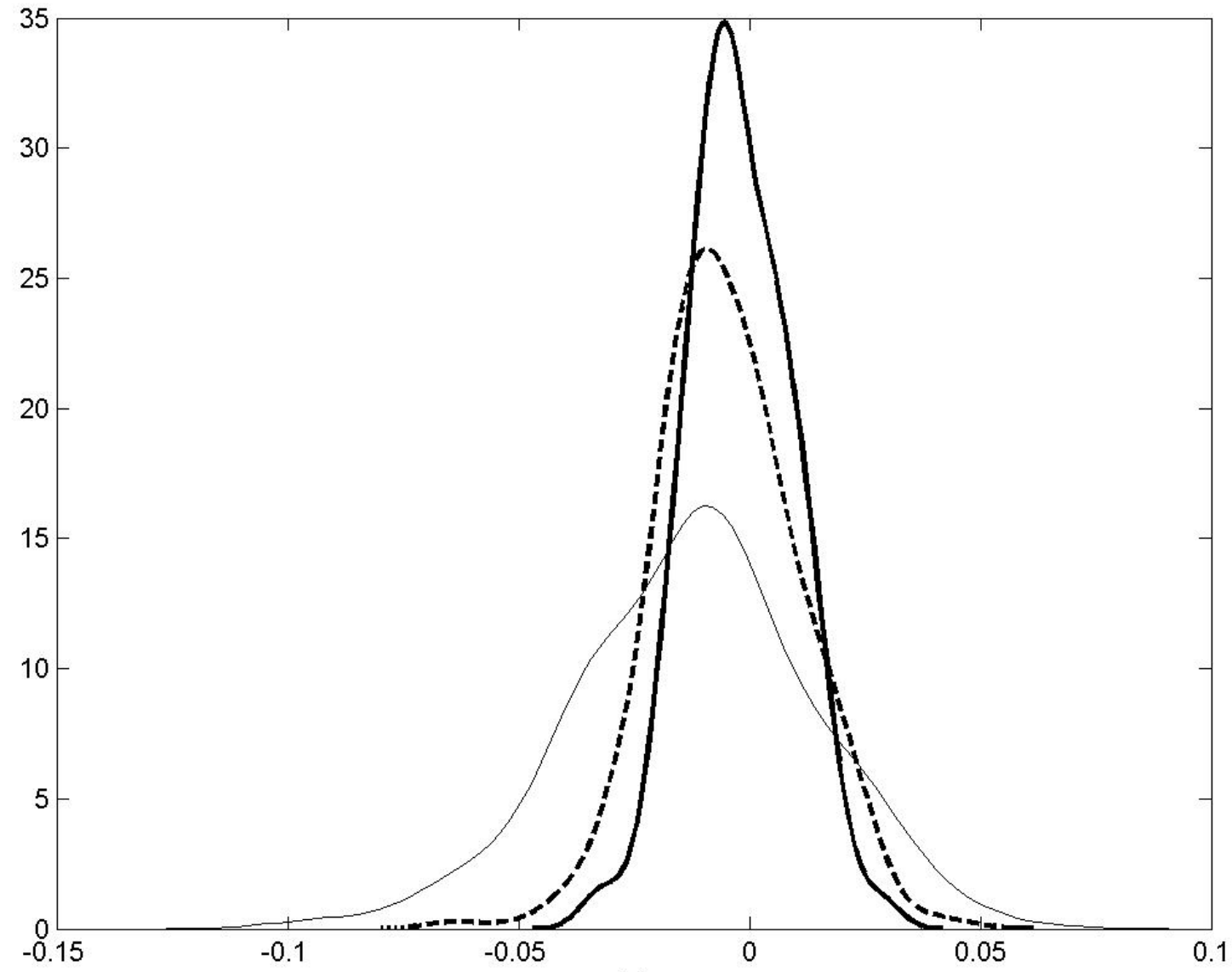

(c)

Figure 5: Distortions of the cross-sectional average of $\operatorname{diag}(\mathcal{R})$ under the correctly specified model. Lines refer to different sample sizes, $T=200$ thin grey line, $T=500$ dashed line and $T=1000$ thick black line. 


\begin{tabular}{lrrrrrrrr}
\hline & \multicolumn{3}{c}{ Misspecified model } & \multicolumn{3}{c}{ Correctly specified model } \\
\hline$T$ & \multicolumn{2}{c}{ OLS } & \multicolumn{2}{c}{ GLS } & \multicolumn{2}{c}{ OLS } & \multicolumn{2}{c}{ GLS } \\
& Mean & Std.dev & Mean & Std.dev & Mean & Std.dev & Mean & Std.dev \\
\hline 200 & 0.440 & 0.326 & 0.440 & 0.324 & 0.443 & 0.331 & 0.442 & 0.328 \\
500 & 0.443 & 0.201 & 0.443 & 0.200 & 0.444 & 0.202 & 0.444 & 0.201 \\
1000 & 0.429 & 0.149 & 0.429 & 0.148 & 0.429 & 0.149 & 0.430 & 0.147 \\
\hline
\end{tabular}

Table 4: Mean and standard deviation of the estimated risk premiums across the 500 replications. The cross-sectional regression adopts an OLS or GLS estimator. The true risk premium corresponds to 0.4167 at the monthly frequency.

in-variable problem. Increasing the sample size, the distortions tend to decrease as well as the dispersion of the estimated risk premiums. There are no differences found between the two estimation approaches, as in the previous case. Finally, as expected, the correctly and misspecified models provide comparable results. We stress that this is a consequence of the data-generating process we follow, in which the risk premium is estimated by looking at the reduced-form betas. In the current data-generating process, with heterogeneous network impacts, the linear factor model provides consistent estimates of the reducedform betas, but does not allow separation of the network and structural elements that affect the betas.

For the first design we provided an example associated with the decomposition of the equally weighted portfolio's idiosyncratic risk, with weights equal to $1 / N$, into the standard component and the network-related component. Here, we repeat the same exercise with two different $\mathcal{R}$ matrices: the first is the one used above, while the second also allows for the presence of negative $\rho_{i}$ coefficients in half of the simulated assets. This second example allows us to highlight the risk absorption effect of the network exposure. While for the first case the results are qualitatively similar to those of the scalar $\rho$ case, when we introduce negative $\rho_{i}$ values, and order assets in descending order of their $\rho_{i}$ values, we note that the introduction of assets with negative $\rho_{i}$ s leads to a moderate decrease of the interconnection impact on the idiosyncratic risk (the fourth component of the variance decomposition). Such an effect could even become negative, thus leading to the absorption of risk by the linked assets, or, in other words, to the amplification of the 


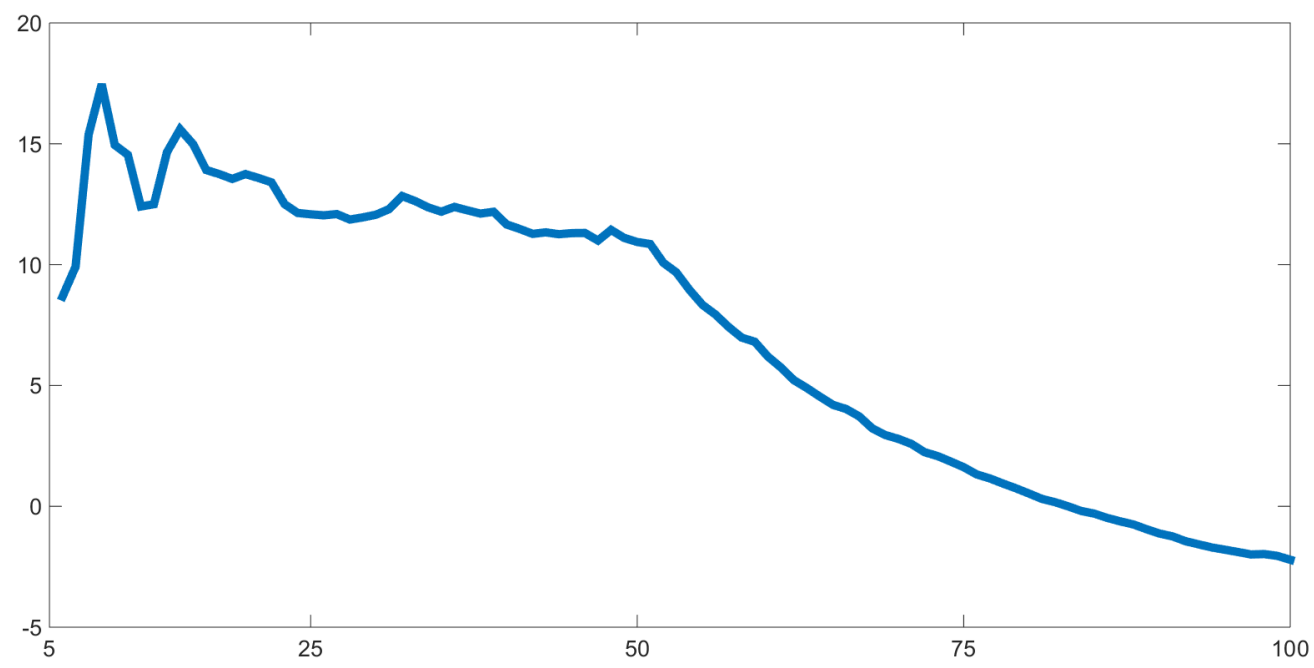

Figure 6: Fourth component of the equally weighted portfolio variance with weights equal to $1 / N$ - impact of interconnections on idiosyncratic risk. Assets in descending order of $\rho_{i}$ values.

diversification benefits. This is evident in Figure 6, where we report the contribution of the fourth component, the network effect on, the idiosyncratic component to the equally weighted portfolio variance, with weights equal to $1 / N$, where the portfolio size increases from 5 to 100 assets, and assets are ranked in descending order of $\rho_{i}$. The last 50 assets have negative network impacts, and the contribution of the interconnections to the idiosyncratic risk becomes negative around asset 80 .

\subsection{Dynamic $W$ and heterogeneous network impact}

In the third simulation design, we combine the heterogeneity in the asset network impact with the time change in the network connections across assets. Now the data-generating process is

$$
\left(I-\mathcal{R} W_{t}\right)\left(R_{t}-\mathbb{E}\left[R_{t}\right]\right)=\bar{\beta} F_{t}+\eta_{t},
$$

Note that, unlike in the previous designs, here the expected returns, conditional on $W_{t}$, are dynamic. To generate a time change in the $W_{t}$ we chose a simple approach, starting from the empirical evidence that the links between assets are persistent; that is, 
we do not have networks that are completely different at times $t$ and $t+1$. Moreover, as commented in the previous section, we do not allow for a change in $W_{t}$ at every $t$ but rather modify $W_{t}$ every $m=20$ observations.

We change $W_{t}$ according to the following scheme: at time 1 we sample $W_{1}$ as in the first design, that is the off-diagonal elements $w_{i, j} \sim \mathcal{B}\left(p_{B}\right)$ with $p_{B}=0.3$; every $m$ observations, each off-diagonal $w_{i, j}$ can take one of only two values, 0 or 1 , and is driven by a Markov chain, with diagonal elements of the transition matrix set as $p_{00}=p_{11}=0.9$. Such a choice ensures persistence in the $W_{t}$ with possibly long-lasting increases/decreases in the associated network density. Finally, we point out that the $W_{t}$ matrices have been normalized with the maximum row normalization.

We now present a number of results obtained from this simulation design. First, we focus on the coefficients $\rho_{i}$. As in the previous case, Figure 7 reports the kernel density for the average of $\operatorname{diag}(\hat{\mathcal{R}})-\operatorname{diag}(\mathcal{R})$ for different sample sizes. We observe a convergence (on average) of the concentrated estimates to the true values with increasing sample sizes, as expected 18

Secondly, we note that, with the data-generating process shown in equation (61), the linear factor model does not estimate the reduced-form betas as, by construction, they are time-varying: $\gamma_{1} \neq\left(I-\mathcal{R} W_{t}\right)^{-1} \beta$. Therefore, to evaluate the distance between those two values, we compute the distortions $\hat{\gamma}_{1}-\left(I-\mathcal{R} W_{t}\right)^{-1} \beta$ and compare them to the distortions under the correctly specified model $\left(I-\hat{\mathcal{R}} W_{t}\right)^{-1} \hat{\beta}-\left(I-\mathcal{R} W_{t}\right)^{-1} \beta$; in both cases, we focus on the cross-sectional averages of the distortions. We collect the results in Table 5. The table shows that the correctly specified model captures the evolution of the reduced-form betas which, we recall, are conditional on the knowledge of the network links. Moreover, the distortions decrease in both mean and dispersion. In contrast, for the misspecified model the distortions do not clearly converge towards the true values but seem to be characterized by an average overestimation of the factor impact.

Finally, we move on to the risk premium estimation and report the results in Table

\footnotetext{
${ }^{18}$ Detailed tables with coefficient-specific results are available upon request.
} 


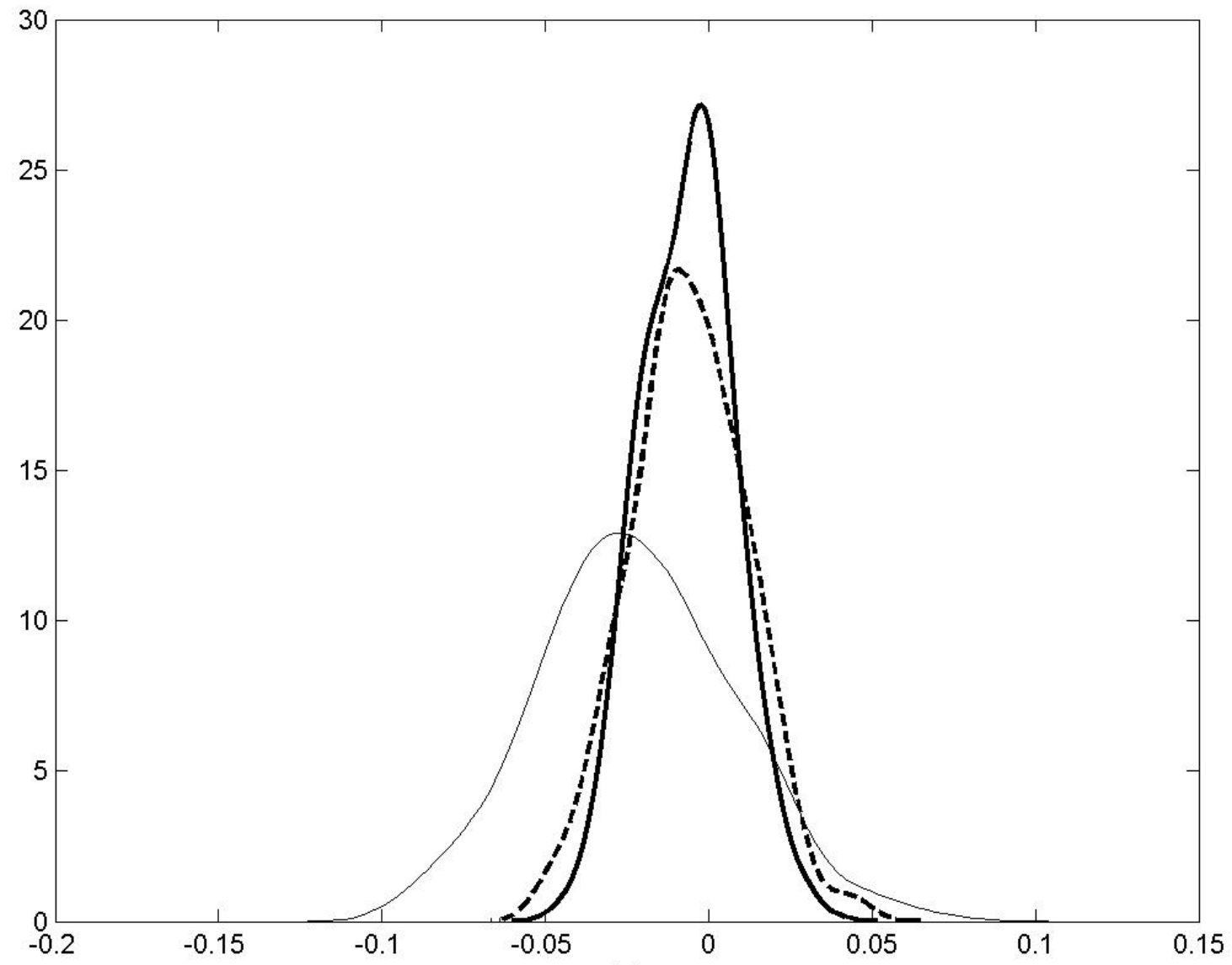

(a)

Figure 7: Distortions of the cross-sectional average of $\operatorname{diag}(\mathcal{R})$ under the correctly specified model. Lines refer to different sample sizes, $T=200$ thin grey line, $T=500$ dashed line and $T=1000$ thick black line.

\begin{tabular}{lrrrr}
\hline$T$ & \multicolumn{2}{c}{ Misspecified model } & \multicolumn{2}{c}{ Correctly specified model } \\
& Mean & Std.dev & Mean & Std.dev \\
\hline 200 & 0.153 & 0.008 & 0.082 & 0.014 \\
500 & 0.205 & 0.003 & 0.055 & 0.008 \\
1000 & 0.194 & 0.002 & 0.040 & 0.006 \\
\hline
\end{tabular}

Table 5: Mean and standard deviation of the cross-sectional averages for the distortions between the estimated betas under the misspecified linear factor model and the reduced-form betas induced by the true model (left-side columns), and between estimated and true reduced-form betas under the correctly specified model (right-side columns). 


\begin{tabular}{lrrrrrrrr}
\hline & \multicolumn{3}{c}{ Misspecified model } & \multicolumn{3}{c}{ Correctly specified model } \\
\hline$T$ & \multicolumn{2}{c}{ OLS } & \multicolumn{2}{c}{ GLS } & \multicolumn{2}{c}{ OLS } & \multicolumn{2}{c}{ GLS } \\
& Mean & Std.dev & Mean & Std.dev & Mean & Std.dev & Mean & Std.dev \\
\hline 200 & 0.413 & 0.316 & 0.412 & 0.314 & 0.417 & 0.323 & 0.415 & 0.355 \\
500 & 0.417 & 0.194 & 0.418 & 0.193 & 0.418 & 0.200 & 0.415 & 0.217 \\
1000 & 0.417 & 0.146 & 0.417 & 0.146 & 0.419 & 0.149 & 0.417 & 0.157 \\
\hline
\end{tabular}

Table 6: Mean and standard deviation of the estimated risk premiums across the 500 replications. The cross-sectional regression adopts an OLS or GLS estimator. The true risk premium corresponds to 0.4167 at the monthly frequency.

6. In this case, the GLS estimator we adopt for the correctly specified model takes into account the known covariance structure across the reduced-form residuals (and thus accounts for an impact of the network links in the estimation of the residual covariance). We first highlight that the OLS and GLS estimates are substantially equivalent. Thus, there is no effect associated with the estimator adopted. Then, we come to the most interesting finding: the risk premiums are very close to the true values for both the correctly and incorrectly specified models, and similarly the risk premium dispersions are very close together under the two estimated models. Distortions were somewhat expected but they have been cancelled out by two elements. The first is the introduction of an averaging across the different matrices $W_{t}$. In fact, under the linear factor model we estimate the betas using the entire sample, which is implicitly affected by the various networks. The reduced-form model estimators are implicitly averaging across the $W_{t}$. The second element is the pattern characterizing the matrix $W_{t}$, which is not exploding. Nevertheless, this second element plays a minor role. Further simulations with different dynamics for the $W_{t}$, introducing a linear or exponential increase in the network density, or a level shift in the network density, confirm the finding 19

\footnotetext{
${ }^{19}$ Additional results are available upon request.
} 


\section{Conclusions}

In this paper we propose a variation of the traditional CAPM in which networks are used to infer the exogenous/lagged and contemporaneous links across assets. We show that this approach allows us to decompose the risk of a single asset (or a portfolio) into four components: (i) the systematic component, (ii) the idiosyncratic component, (iii) the impact of the asset interconnections on the systematic risk component, that is, the contribution of network exposure to the systematic risk component, and (iv) the effect of interconnections on the effect of idiosyncratic risk on the systematic risk component, that is, the amplification of idiosyncratic risks that generates systematic/non-diversifiable risk. Our approach also allows us to decompose the risk premium component of returns into three components: the risk premiums associated with (i) common factor exposures, (ii) the impact of asset connections on common factors and (iii) the amplification effects of idiosyncratic risk. The simulation analysis we perform shows that the new model we propose can be used to better understand the different components of systematic risk and volatilities and analyse the causes of systematic risk. Moreover, the simulation allows us to disentangle the error estimation of linear factor models that ignore the presence of network connections. In particular, we show that the residual correlations start drifting away from zero if network connectedness is ignored in the model estimation.

This new model is relevant for policy makers and regulators, since they need to be aware of the implications of the different possible policy choices on network connections and their effects on equilibrium stock returns and volatilities, as well as for investors and other market participants, since they need to understand whether and to what degree network connectivity has an impact on risk premiums, volatilities and spillovers between markets. The model could be analysed not only through simulations but also using real data. We plan to work on this in our future research. 


\section{References}

[1] Abbassi,P., C. Brownlees, C. Hans and N. Podlich, 2014, Bank Interconnectedness. What Determines the Links? Working paper.

[2] Abreu M., H.L.F. de Groot and R.J.G.M. Florax, 2005, Space and growth: a survey of empirical evidence and methods, Region et Developpement, 21, 13-44.

[3] Acemoglu D., V.M. Carvalho, A. Ozdaglar and A. Tahbaz-Salehi, 2012, The Network Origins of Aggregate Fluctuations, Econometrica, 80, 1977-2016.

[4] Ahern K., 2013, Network centrality and the cross section of stock returns. Working paper, University of Southern California.

[5] Allen F., and A. Babus, 2009, Networks in Finance, in The Network Challenge, edited by P. Kleindorfer and J. Wind, Philadelphia, PA: Wharton School Publishing, 367382.

[6] Allen F., and D. Gale, 2000, Financial Contagion, Journal of Political Economy, 108, $1-33$.

[7] Alves I., S. Ferrari, P. Franchini, J.C. Heam, P. Jurca, S. Langfield, S. Laviola, F. Liedorp, A. Sanchez, S. Tavolaro and G. Vuillemey, 2013, The structure and resilience of the European interbank market, ESRB Occasional Paper No.3.

[8] Ang, Andrew, R.J. Hodrick, Yuhang Xing and Xiaoyan Zhang, 2006, The cross section of volatility and expected returns, The Journal of Finance, 61, 259-299.

[9] Anselin L., 1988, Spatial Econometrics: methods and models, Boston: Kluwer Academic Publishers.

[10] Asgharian H., W. Hess and L. Liu, 2013, A spatial analysis of international stock market linkages, Journal of Banking and Finance, 37, 4738-4754. 
[11] Barigozzi M., and C. Brownlees, 2014, NETS, Network Estimation for Time Series. Working paper.

[12] Bianchi D., M. Billio, R. Casarin, M. Guidolin, 2015, Modeling Contagion and Systemic Risk 2015. Working paper.

[13] Billio M., M. Getmansky, A. Lo and L. Pelizzon, 2012, Econometric Measures of Connectedness and Systematic Risk in the Finance and Insurance Sectors, Journal of Financial Economics, 104, 535-559.

[14] Billio M., D. Gray, M. Getmansky, A.Lo, R. Merton and L.Pelizzon, 2014, Sovereign, bank and insurance credit spreads: Connectedness and system networks. Working paper.

[15] Billio M., M. Caporin, L. Frattarolo and L. Pelizzon, 2015, Network banks exposures and variance spillovers in the Euro area. Working Paper

[16] Black F., M.C. Jensen and M. Scholes, 1972, The capital asset pricing model: Some empirical tests, in Studies in the Theory of Capital Markets, Praeger, edited by Michael C. Jensen, 79-121.

[17] Blasques F., S.J. Koopman, A. Lucas and J. Schaumburg, 2014[2013 cited on page 28 of pdf], Spillover Dynamics for Systemic Risk Measurement Using Spatial Financial Time Series Models. SYRTO Working Paper 16.

[18] Branger N., P. Konermann, C. Meinerding and C. Schlag, 2014, Network Connectivity, Mutually Exciting Jumps and Equilibrium Asset Prices. Working paper.

[19] Buraschi A., and P. Porchia, 2013, Dynamic Networks and Asset Pricing. Working paper.

[20] Carhart M., 1997, On the persistence of mutual fund performance, Journal of Finance, $52,1-133$. 
[21] Cifuentes R., G. Ferrucci and H.S. Shin, 2005, Liquidity risk and contagion, Journal of the European Economic Association, 3, 556-566.

[22] Cochrane John H., 2011, Presidential address: Discount rates, Journal of Finance, 66, 1047-1108.

[23] Dasgupta A., 2004, Financial contagion through capital connections: a model of the origin and spread of bank panics, Journal of the European Economic Association, 2(6), 1049-1084. corrected order

[24] Degryse H., and G. Nguyen, 2007, Interbank exposures: an empirical examination of systemic risk in the Belgian banking system, International Journal of Central Banking, 3(2), 123-171.

[25] Diebold F., and K. Yilmaz, 2014, On the Network Topology of Variance Decompositions: Measuring the Connectedness of Financial Firms, Journal of Econometrics, $182,119-134$.

[26] Diebold F., and K. Yilmaz, 2015, Financial and Macroeconomic Connectedness: A Network Approach to Measurement and Monitoring, Oxford University Press.

[27] Dupor B., 1999, Aggregation and Irrelevance in Multi-Sector Models, Journal of Monetary Economics, 43, 391-409.

[28] Elhorst J.P., 2003, Specification and estimation of spatial panel data models, International Regional Science Review, 26, 244-268.

[29] Elsinger H., A. Lehar and M. Summer, 2006, Using market information for banking system risk assessment, International Journal of Central Banking, 2(1), 137-165.

[30] Fama E., and K.R. French, 1993, Common risk factors in the returns of stocks and bonds, Journal of Financial Economics, 33, 3-53.

[31] Fama E., and K.R. French, 1995, Size and book-to-market factors in earnings and returns, Journal of Finance, 50(1), 131-155. 
[32] Fama E., and J.D. MacBeth, 1973, Risk, return and equilibrium: Empirical tests, Journal of Political Economy, 81, 607-636.

[33] Fernandez V., 2011, Spatial linkages in international financial markets, Quantitative Finance 11, 237-245.

[34] Furfine C., 2003, Interbank Exposures: Quantifying the Risk of Contagion, Journal of Money, Credit and Banking, 35(1), 111-128.

reordered according to increasing year of publication

[35] Hautsch N., J. Schaumburg and M. Schienle, 2012, Financial Network Systemic Risk Contributions, Collaborative Research Center 649, Discussion Paper 2012-053.

[36] Hautsch N., J. Schaumburg and M. Schienle, 2013, Forecasting systemic impact in financial networks, International Journal of Forecasting, 30, 781-794.

[37] Horvath M., 1998, Cyclicality and Sectoral Linkages: Aggregate Fluctuations from Sectoral Shocks, Review of Economic Dynamics, 1, 781-808.

[38] Katz L., 1953, A new status index derived from sociometric analysis, Psychometrika, $18,39-43$.

[39] Keiler S., and A. Eder, 2013, CDS spreads and systemic risk, a spatial econometric approach, Deutsche Bundesbank Discussion Paper, No. 1/2013.

[40] Kelly B.T., H.N. Lustig and S.V. Nieuwerburgh, 2013, Firm Volatility in Granular Networks, Fama-Miller working paper, Chicago Booth Research Paper No. 12-56.

[41] Kou S., X. Peng and H. Zhong, 2016, Asset pricing with spatial interaction, Management Science, manuscript MS-15-01029.R1.

[42] Lagunoff R., and S. Schref, 2001, A model of financial fragility, Journal of Economic Theory, 99, 220-264. 
[43] Leitner Y., 2005, Financial networks: contagion, commitment and private sector bailouts, Journal of Finance, 60, 2925-2953.

[44] LeSage, James P., 1999, Spatial Econometrics, Morgantown: Regional Research Institute, University of West Virginia.

[45] LeSage J., and R.K. Pace, 2009, Introduction to spatial econometrics, Hall/CRC, London.

[46] Lintner J., 1965a, The valuation of risk assets and the selection of risky investments in stock portfolios and capital budgets, Review of Economics and Statistics, 47, 13-37.

[47] Lintner J., 1965b, Security prices, risk and maximal gains from diversification, Journal of Finance, 20, 587-615.

[48] Lucas R., 1977, Understanding Business Cycles, Carnegie-Rochester Conference Series on Public Policy, 5, 7-29.

[49] Markowitz H., 1952, Portfolio selection, Journal of Finance, 7, 77-91.

[50] Mistrulli P., 2011, Assessing financial contagion in the interbank market: maximum entropy versus observed interbank lending patterns, Journal of Banking \& Finance, $35(5), 1114-1127$.

[51] Mossin J., 1966, Equilibrium in a capital asset market, Econometrica, 35, 768-783.

[52] Ozdagli A., M. Weber, 2015, Monetary Policy Through Production Networks: Evidence from the Stock Market, Working Paper.

[53] Ozsoylev HN. and J. Walden, 2011, Network models and financial stability, Journal of Economic Theory, 146(6), 2252-2280.

[54] Ross S.A., 1976, The arbitrage theory of capital asset pricing, Journal of Economic Theory, 13, 341-360. 
[55] Shanken, J., 1992, On the estimation of beta pricing models, Review of Financial Studies 5, 1-34.

[56] Sharpe W.F., 1964, Capital Asset Prices: A Theory of Market Equilibrium Under Conditions of Risk, Journal of Finance, 19, 425-442.

[57] Shea J., 2002, Complementarities and Comovements, Journal of Money, Credit, and Banking, 34, 412-433.

[58] Upper C., 2011, Simulation methods to assess the danger of contagion in interbank networks, Journal of financial stability,7, 111-125.

[59] Upper C., and A. Worms, 2004, Estimating bilateral exposures in the German interbank market: Is there a danger of contagion?, European Economic Review, 48(4), 827-849.

[60] Van Lelyveld I., and F. Liedorp, 2006, Interbank contagion in the Dutch banking sector: a sensitivity analysis, International Journal of Central Banking, 2, 99-134.

[61] Wagner H., 2010, The causes of the recent financial crisis and the role of central banks in avoiding the next one, International Economics and Economic Policy, 7(1), 63-82.

[62] Wells S., 2004, Financial interlinkages in the United Kingdom, Âôs interbank market and the risk of contagion. Working Paper, 230, Bank of England. 


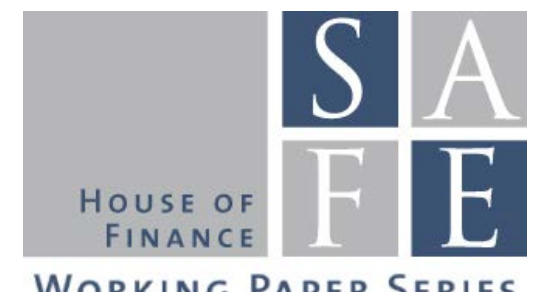

WORKING PAPER SERIES

\section{Recent Issues}

No. 165 Giovani Bonaccolto, Massimiliano Caporin, Roberto Panzica

No. 164 Raimond Maurer, Olivia S. Mitchell, Ralph Rogalla, Tatjana Schimetschek

No. 163 Giuliano Curatola, Michael Donadelli, Patrick Grüning

No. 162 Gabriele Camera, Alessandro Gioffré

No. 161 Tobin Hanspal

No. 160 Domenico Rocco Cambrea, Stefano Colonnello, Giuliano Curatola, Giulia Fantini

No. 159 Monica Billio, Michael Donadelli, Antonio Paradiso, Max Riedel

No. 158 Michael Donadelli, Renatas Kizys, Max Riedel

No. 157 Steffen Meyer, Linda Urban, Sophie Ahlswede

No. 156 Reint Gropp, Thomas Mosk, Steven Ongena, Carlo Wix

No. 155 Vahid Saadi

No. 154 Brigitte Haar

No. 153 Julia Hirsch, Uwe Walz
Estimation and model-based combination of causality networks

Optimal Social Security Claiming Behavior under Lump Sum Incentives: Theory and Evidence

Technology Trade with Asymmetric Tax Regimes and Heterogeneous Labor Markets: Implications for Macro Quantities and Asset Prices

Asymmetric Social Norms

The Effect of Personal Financing Disruptions on Entrepreneurship

Abandon Ship: Inside Debt and Risk-Taking Incentives in Bad Times

Which Market Integration Measure?

Globally Dangerous Diseases: Bad News for Main Street, Good News for Wall Street?

Does feedback on personal investment success help?

Bank Response To Higher Capital Requirements: Evidence From A QuasiNatural Experiment

Mortgage Supply and the US Housing Boom: The Role of the Community Reinvestment Act

Shareholder Wealth vs. Stakeholder interests? Evidence from Code Compliance under the German Corporate Governance Code

The Financing Dynamics of Newly Founded Firms 